\title{
The kleineWeltentdecker App - A Smartphone-Based Developmental Diary
}

\author{
Moritz M. Daum ${ }^{1 *}$, Marco Bleiker ${ }^{1}$, Stephanie Wermelinger ${ }^{1}$, Ira Kurthen ${ }^{1}$, Laura \\ Maffongelli $^{2}$, Katharina Antognini ${ }^{3}$, Miriam Beisert ${ }^{1}$, and Anja Gampe ${ }^{1,4}$ \\ ${ }^{1}$ University of Zurich, Switzerland \\ ${ }^{2}$ Johannes Gutenberg University Mainz, Germany \\ ${ }^{3}$ University of Applied Sciences in Special Needs Education, Zurich, Switzerland \\ ${ }^{4}$ University of Duisburg-Essen, Germany
}

This version of the article has been accepted for publication at Behavior Research Methods, after peer review but is not the Version of Record and does not reflect post-acceptance improvements, or any corrections. The Version of Record will available online at: http://dx.doi.org/10.3758/s13428-021-01755-7.

Date: 01/February/2022

\section{Author Note}

Correspondence concerning this article should be addressed to Moritz M. Daum https://orcid.org/0000-0002-4032-4574, University of Zurich, Department of Psychology and Jacobs Center for Productive Youth Development, Developmental Psychology: Infancy and Childhood, Binzmuehlestrasse 14, Box 21, CH-8050 Zurich, Switzerland. Phone: +41 4463574 80, E-mail: moritz.daum@uzh.ch 


\begin{abstract}
Today, a vast number of tools exist to measure development in early childhood in a variety of domains such as cognition, language, or motor, cognition. These tools vary in different aspects. Either, children are examined by a trained experimenter, or caregivers fill out questionnaires. The tools are applied in the controlled setting of a laboratory or in the children's natural environment. While these tools provide a detailed picture of the current state of children's development, they are at the same time subject to several constraints. Furthermore, the measurement of an individual child's change of different skills over time requires not only one measurement but high-density longitudinal assessments. These assessments are time-consuming, and the breadth of developmental domains assessed remains limited. In this paper, we present a novel tool to assess the development of skills in different domains, a smartphone-based developmental diary app (the kleineWeltentdecker App, henceforth referred to as the APP ${ }^{1}$ ). By using the APP, caregivers can track changes in their children's skills during development. Here, we report the construction and validation of the questionnaires embedded in the APP as well as the technical details. Empirical validations with children of different age groups confirmed the robustness of the different measures implemented in the APP. In addition, we report preliminary findings, for example, on children's communicative development by using existing APP data. This substantiates the validity of the assessment. With the APP, we put a portable tool for the longitudinal documentation of individual children's development in every caregiver's pocket, worldwide.

Keywords: ambulatory assessment, experience sampling, longitudinal research design, smartphone application
\end{abstract}

\footnotetext{
${ }^{1}$ The German expression "kleine Weltentdecker" can be translated as "young world explorers".
} 


\section{The kleineWeltentdecker App - A Smartphone-Based Developmental Diary}

\section{Challenges in the Longitudinal Assessment of Development}

The measurement of developmental change is challenging. Our current

3 knowledge about children's development results to a large extent from cross-sectional

4 studies. Mostly, different individuals of different ages are tested within a narrow time

5 window. This approach is vital for the assessment of age differences, but it only

6 provides a static picture of current developmental states. As a result, a large amount of

7 research in developmental psychology is dedicated to the description of children's

8 behaviour at different ages. It has therefore become somewhat conventional to describe

9 the earliest manifestations of particular abilities (Adolph et al., 2008). However, already

10 Vygotsky raised the concern that a cross-sectional approach primarily focuses on

11 age-dependent and stable endpoints in development (Vygotsky, 1978). Similarly,

12 Adolph and colleagues stated that this kind of research has resulted in "a gallery of

13 before and after snapshots, studio portraits of newborns, and fossilised milestones"

14 (Adolph et al., 2008, p. 527). With these static developmental pictures, little can be

15 learned about developmental processes.

16 To a certain degree, this shortcoming is compensated for by longitudinal research

17 paradigms. Here, the same individuals are tested multiple times at predefined

18 measurement points, for example, every month or every year. This approach provides

19 information about individual developmental trajectories by relating early and later

20 developing skills. However, this assumed "gold standard" approach has likewise

21 disadvantages. First, it remains unknown what happens between the different

22 measurement points. According to Adolph and colleagues, "sampling rates typically

23 used by developmental researchers may be inadequate to accurately depict patterns of

24 variability and the shape of developmental change" (Adolph et al., 2008, p. 527). That

25 is, when the sampling rate chosen is too low, it does not allow to identify whether a

26 developmental trajectory reflects a smooth and monotonic improvement, a non-linear

27 trend, or an accelerating or decelerating transformation. Second, even on a small scale,

28 longitudinal studies are often highly resource intensive. They require an extensive 
29 amount of human and financial resources, and often a substantial amount of time.

30 Third, the measurement points are usually determined based on the mean age at which

31 certain developmental milestones are expected to be reached. This limits the validity of

32 standard longitudinal research paradigms because the assumed mean age and the

33 accordingly determined measurement point do not necessarily reflect a single

34 individual's development (Hamaker, 2012). In addition, the actual moment in which a

35 developmental change occurs is not captured with predefined measurement points.

36 To overcome this limitation, we present a new smartphone-based developmental

37 diary approach that adapts the Age-of-Attainment (AoA) method (e.g., W. O. Eaton

38 et al., 2014). The AoA method has its roots in event-centred approaches (e.g.,

39 Campbell \& Weech, 1941; Wohlwill, 1973). It does not measure developmental

40 processes by the presence or absence of a developmental milestone, for example,

41 whether or not a 12-month-old already walks independently. Rather, it helps to identify

42 the point in time of the emergence of the skill. This allows researchers to capture the

43 individual age differences at which children reach a specific developmental milestone. As

44 a result, the AoA method helps to shift age from being a predictor of other variables to

45 being the outcome explained by those other variables (Wohlwill, 1973). The age at

46 which children first reach a specific developmental milestone (e.g., independent walking)

47 shows substantial inter-individual variability. Capturing this variability may reveal

48 information about underlying developmental processes, for instance by informing about

49 how skills acquired early relate to later ones (Bornstein et al., 2013, Dinehart \& Manfra,

50 2013). Determining the AoA requires behavioural observation that is of higher

51 frequency than the usual applied yearly or monthly observations (optimally, a 24/7

52 tracking of a child's development). For feasibility reasons, this requires outsourcing data

53 collection from the controlled environment of a laboratory to the home environment of

54 the children and their caregivers. Current technological developments, such as the

55 widespread availability of smartphones, have the potential to overcome the limitations

56 developmental research was facing so far and to facilitate the collection of

57 comprehensive AoA data. 


\section{Skills do Not Develop Independently of Each Other}

In the past, various researchers have described how to implement designs with

60 multiple outcome measures (Aslin, 2007, LoBue \& Adolph, 2019; LoBue et al., 2020;

61 Morris et al., 2006). With the present APP, we aim to expand this view by focusing on

62 the second methodological and theoretical challenge of developmental research: Skills

63 do not develop in isolation. Neither, they develop independently from each other nor

64 independently of the environmental context, which also changes at the micro, meso, and

65 macro levels (Bronfenbrenner, 1992). On the contrary, when a particular skill in one

66 domain occurs or changes, skills in other domains often do not remain unaffected (e.g.,

67 Smith \& Thelen, 2003).

68 Let's exemplify this with the development of basic motor skills: Motor

69 development results from the co-occurrence and interactions of basic maturation

70 processes such as the increased myelinisation of the cortical-spinal tract McGraw, 1943 .

71 Zelazo, 1998, other physiological systems (muscle strength and the ability to balance

72 Adolph et al., 2003, Spencer et al., 2000), cognitive and perceptual skills,

73 social-emotional change (e.g., the motivation to move independently), experience

74 (adequate opportunities to practice the emerging skill), which are often influenced by

75 cultural and historical differences in child-rearing practices Adolph and Hoch, 2019.

76 Vice versa, the development of motor skills is strongly influenced and refined by

77 perceptual, cognitive, motivational skills as well as by cultural and historical differences

78 in child-rearing practices (Adolph \& Hoch, 2019). And vice versa, the acquisition of

79 new motor skills lays the cornerstone for the emergence and refinement of skills in other

80 domains (Soska et al., 2015, for overviews, see Campos et al., 2000 Gredebäck et al.,

81 2021). For example, changes in locomotion result in changes in perception: Crawling

82 infants' look down at the floor to a great extent. In contrast, walking infants direct

83 their gaze at their caregivers and objects in the environment (Kretch et al., 2014).

84 Furthermore, locomotion influences infants' cognitive skills (Campos et al., 2000), such

85 as their mental rotation of objects: Crawling infants show better mental rotation than

86 non-crawling infants (Schwarzer et al., 2013). Mental rotation is further positively 
87 influenced by the infants' general motor experience (Frick \& Wang, 2013, Möhring \&

88 Frick, 2013). Also, fine-motor skills (Dinehart \& Manfra, 2013) and early action

89 experiences (Bornstein et al., 2013) are significantly related to later academic

90 achievement. Concerning the cultural and historical context, it has been shown that the

91 position in which children sleep (supine or prone) has an impact on the age of

92 acquisition several of motor milestones. Compared to supine sleepers, prone sleepers

93 start earlier rolling prone to supine, tripod sitting, creeping, crawling, and pulling to

94 stand (Davis et al., 1998). The American Academy of Pediatrics recommended in 1992

95 that infants should be placed on their side or back for sleep (Pediatrics, 1992) to reduce

96 the incidence of sudden infant death syndrome. With this intervention, the percentage

97 of infants sleeping prone has decreased and, accordingly, the age when different motor

98 skills are acquired has increased. This shows how the context in which children grow up

99 provides different opportunities resulting in different developmental trajectories.

100 While knowledge about specific interrelations such as the ones just reported is

101 increasing, the assessment of the development of the interrelations between skills in

102 different domains and, in particular, their dynamic interaction over time remains

103 limited. The developmental diary approach presented here implements the following

104 features: It includes the development of skills in different domains (cognition, language,

105 motor, and social and emotional skills). The temporal assessment is shorter compared

106 to a large number of longitudinal studies. It relates the development of these different

107 domains to each other. Finally, it considers contextual factors such as the language(s)

108 spoken by the child and the caregivers, and the caregivers' cultural, educational, and

109 economic background.

\section{Goals of the kleineWeltentdecker App (APP)}

111 To address these challenges, we developed the kleineWeltentdecker App

112 (henceforth referred to as the APP), a smartphone-based digital developmental diary

113 application. With the APP, we provide a tool to the caregivers to document the

114 development of their children from age 0 to 6 . At the same time, caregivers share the 
115 data of their children's development anonymously with our research unit (see also

116 section Data Security and Data Protection. With this participatory science approach

117 ("caregiver-as-a-researcher"), the APP allows acquiring and analysing longitudinal data

118 at a relatively high temporal resolution optimally at the exact moment when a

119 developmental change occurs. The following three goals drove the development of this

120 research tool and its related research.

121 Goal 1: Establish a Comprehensive Data Set of Child Development From

122 Age 0 to 6, Within and Across Individuals

123 Given the ubiquity of smartphones worldwide, the range of use of the APP is not

124 limited to specific regions or countries. This aspect facilitates the analysis of the

125 variability of behaviour and its development regarding contextual aspects such as

126 culture, SES, language background, and many other demographic and family factors.

127 The acquired data are therefore subject to analyses for the following major purposes:

128 1) It allows an in-depth analysis of the individual developmental trajectories of major

129 developmental domains. 2) It allows analysis of the dynamically changing interrelations

130 and inter-dependencies of the development in the individual domains. With this

131 approach, developmental trajectories can be compared within and between individuals,

132 or within and between cultures, which helps to identify developmental specificities and

133 universals.

\section{Goal 2: Account for the Variability on Development Across Cultures}

135 One issue of increasing importance in developmental science is the variability in

136 children's development. Previous research on psychology in general and in

137 developmental psychology in particular is based on data from WEIRD (Western,

138 Educated, Industrialised, Rich, and Democratic) populations (e.g., Nielsen et al., 2017).

139 There is a growing number or researchers who argue that this approach undermines the

140 variability of behaviour and development across the globe. Henrich et al. (2010b) state

141 that their "findings suggest that members of WEIRD societies, including young

142 children, are among the least representative populations one could find for generalising 
143 about humans" (p.61). A bias towards WEIRD samples may result in that findings,

144 which are specific to a particular culture, are falsely being interpreted as universal traits

145 (Henrich et al., 2010a; Nielsen et al., 2017). Accordingly, the second goal of the APP is

146 to provide a tool that is not (or at least much less) restricted to the collection of data

147 within a narrow range of participants but is - optimally - available world wide. In a first

148 step, we implemented the APP in four different languages: British English, French,

149 German, and Italian.

150 We are aware that, currently, the APP asks caregivers about their children's

151 skills in a fixed and to some extent "WEIRD"-based order and children from different

152 cultures may develop in a different order. However, the current approach will help to

153 identify commonalities and differences between cultures and will be helpful to identify

154 developmental sequences that differ from norms based on WEIRD societies.

\section{Goal 3: Outsourcing of Data Collection}

1576 requires an enormous effort and is resource-intensive. With the APP, data collection

158 is outsourced to the caregivers of the child. This approach does not come without

159 challenges that will be addressed in greater detail in the section Goals and Challenges

160 in the Discussion below. Caregivers experience their children's behaviour in more

161 instances and more varied situations than a laboratory setting can establish. These

162 different contexts might support the observation of the emergence a new skill. Several

163 features were implemented to facilitate caregiver evaluation: Caregivers receive

164 packages of questions that fit the child's current age range in which the developmental

165 steps usually occur. The questions are enriched with information about possible

166 variations of the observable behaviour to facilitate answering the questions. Besides this

167 standard procedure, it is of course possible to answer questions that are not in these

168 packages. Like this, the predefined selection of questions, which is based on the mean

169 age of development, does not restrict answering questions that are outside of this age

170 window. Further, the questions are complemented with additional information about

171 the particular behaviour and how it is integrated into children's development from a 
172 broader perspective. This helps caregivers to evaluate whether or not their child already

173 shows a certain skill or not.

174 To sum up, with the APP, caregivers are provided with a scientifically

175 substantiated tool to document the development of their children between birth and the

176 age of 6 years. It is designed to be intuitive and easy to use to facilitate continued and

177 sustained documentation of development. The developmental steps and milestones are

178 scientifically corroborated and have been tested for reliability by comparing them to

179 standard instruments (see section Psychometric Properties of Scale, below).

180 In the following sections, we describe the different APP scales in more detail. We

181 will explain the major participant target group of the APP, the construction of the

182 different scales, ethics and data security, technical specifications and provide details on

183 the psychometric properties of the APP.

\section{Scale Protocol}

\section{Participants: Target Group}

The target group of the APP are caregivers of children between 0 and 6 years.

187 We conducted a survey among 799 Swiss caregivers who already participated with their

188 children in one or more studies of our research unit. The results showed that $>85 \%$ of

189 the caregivers would like to use a digital developmental diary app and $>95 \%$ of these

190 would agree to share the data with the research unit. In general, caregivers seem to be

191 open-minded to modern media and a substantial number of caregivers is willing to use

192 the APP and share the collected data increasing the potential for acquiring data from a

193 sample large enough to make reliable conclusions.

\section{Content: Domains and Items}

195 The questions implemented in the APP target the main domains in early

196 childhood development: cognitive, language, motor, and social-emotional skills. To

197 obtain a comprehensive picture about the context of each individual child's

198 development, questions about caregiver education, country of birth, family constellation, 
language exposure at home and in childcare, etc. are included. An overview of all questions is available in the Open Science Framework (OSF; https://osf.io/ar7xp/).

\section{Construction of Items}

To be included in the APP, items had to fulfill two main characteristics: On the

203 one hand, developmental skills assessed within the APP need to be scientifically

204 relevant. That is, the skills have been documented in scientific papers on infant and

205 child development or are included in diagnostic tools to assess the development of the

206 skills of a child at a given age. On the other hand, the APP has to account for the fact

207 that the questions are not answered by trained experts but by caregivers who might not

208 be familiar with the jargon of developmental psychology. Accordingly, the assessment of

209 skills needs to be tailored in a way that it can easily yet still reliably be performed by

210 the caregivers, independent of their language skills and educational background. That

211 is, for all scales, the items were formulated so that they a) are easy to understand and

212 imply face validity, b) refer to the child's observable behaviour and do not require

213 implicit measurements, c) can be clearly, objectively, and reliably answered by the

214 caregivers' observation alone (avoiding sophisticated measurement techniques), d) refer

215 to materials which can be found in a usual household, and e) still ensure scientific

216 precision.

217 The construction procedure included the following three steps: 1) We started

218 with a comprehensive literature search collecting skills that typically develop in the first

2196 years of life in the domains of cognitive, language, motor, and social-emotional

220 development. 2) All skills identified were evaluated with regard to whether it was

221 possible to formulate a question and corresponding answer options that are scientifically

222 relevant, precise, and unambiguous and at the same time feasible and understandable

223 for laypeople. This initial collection of potential items comprised 34 items on cognitive

224 development, 194 items on motor development, and 245 items on language development.

225 3) From these preliminary items, we created a first set of 'pre'-questionnaires and asked 226 caregivers of children between 3 and 78 months $\left(n=1397 ; n_{\text {girls }}=657, n_{\text {boys }}=739\right.$, 


\section{Table 1}

Number of Items per Domain that were Included in the Final Version of the APP.

\begin{tabular}{lr}
\hline Domain & Number of Items \\
\hline Cognition & 34 \\
Language Skills (Syntax, Grammar) & 157 \\
Motor & 176 \\
Social / Emotional & 151 \\
Demographics & 24 \\
Total & 630 \\
\hline
\end{tabular}

$n_{\text {other }}=1, M_{\text {age }}=464$ days, $S E=526$ days) to fill them out. Caregivers additionally

228 provided feedback on whether a particular item was easy or difficult to assess or

229 ambiguous in its formulation. Based on this feedback, 17 items were excluded from the

230 final APP scale. Including the items of the social-emotional scale that were adapted

231 from existing scales (e.g., the Infant Behavior Questionnaire - Revised (IBQ-R),

232 Gartstein \& Rothbart, 2003 , see section "Social-Emotional Scale", below), this process

233 resulted in a total number of 630 items, see Table 1. The particular construction of the

234 items in the four domains (Scales) is described in more detail in the following sections.

\section{Cognitive Scale}

236 The items of the cognitive scale are grouped according to the following

237 constructs: sensori-motor development, problem solving, and numerical and categorical

238 knowledge. The 19 sensori-motor items include questions on children's object

239 exploration and manipulation, reaching, attention, pointing, imitation, and pretend

240 play. The 9 problem-solving items assess children's object permanence, means-end

241 behaviour, memory, and mastery of new problems. The 6 items on numerical and

242 categorical knowledge include questions on children's counting abilities, colour naming

243 skills, and knowledge about object sizes and physical laws. For item construction, the

244 cognitive scales of existing instruments such as Bayley Scales of Infant Development

245 (Bayley, 1993), the Intelligence and Development Scales - Preschool (IDS-P; Grob 
246 et al., 2013), and the Griffiths Scales of Childhood Development (Green et al., 2016)

247 were screened and served as a basis for item selection. One item was created by the

248 authors. It describes a behaviour that is commonly observed by caregivers and

249 considered as a milestone in development but was not found in any developmental scale

250 (CG34: "Can your child tie his/her own shoelaces?"). Each item sketches a concrete

251 behaviour or instructs to provoke a certain behaviour. For details on answer options

252 and examples of items see Appendix

\section{Language Scale}

254 The following skills were implemented in the language scale: early pre-verbal,

255 morphological, and syntactical skills as well as pragmatic skills. The 16 early pre-verbal

256 skills include cooing, babbling, and the production of gestures such as pointing. The

257 morphology scale consists of 23 items. It includes the flexion of adjectives, nouns for

258 plural, and verbs for past and present tense. It further includes fusion of articles and

259 pronouns or prepositions. The syntax scale comprises 65 items on the combination of

260 clauses using conjunctions and relative clauses, Wh-questions, indirect speech and

261 conditionals. To assess pragmatics skills, we implemented the Orion's Pragmatic

262 Language Skills Questionnaire (e.g., Ghahari et al., 2017), which assesses nonverbal

263 communication, language production, conversational skills like topic maintenance and

264 turn taking, speech conventions, and peer skills in 53 items. For all morphological and

265 syntactical skills, we created prototypical sentences in which the target morphological

266 flexion or syntactic construction word were highlighted. The sentences include

267 every-day topics like caregivers working, children visiting playgrounds, reading books

268 etc. The words used in the prototypical sentences to express these topics are all early

269 acquired (in the first 2-3 years) by children as cross-validated with the MacArthur Bates

270 Communicative Developmental Inventories (Fenson et al., 2007). For details on answer

271 options and examples of items see Appendix. 


\section{Motor Scale}

The motor scale includes fine- and gross-motor skills. The 78 fine-motor items

274 include visual-motor integration, grasping, and graphomotorics. The 98 gross-motor

275 items include stationary motor skills, locomotion, and object manipulation. Item

276 construction was geared towards existing scales such as the Peabody Developmental

277 Motor Scales: Second Edition (PDMS-2; Folio \& Fewell, 2000) or the Bailey Scales of

278 Infant Development: Second Edition (BSID-ii; Bayley, 1993). Scales were screened and

279 served as a basis for the decision which items to include in the diary. For all identified

280 motor skills, we created items that describe important motor milestones. For details on

281 answer options and examples of items see Appendix.

\section{Social-Emotional Scale}

The social-emotional scale includes measures of infants' and children's

284 temperament and attention as well as their Theory of Mind (ToM). Child temperament

285 is considered stable over time and a personality trait (Goldsmith \& Campos, 1982 ,

286 Rothbart, 1981; Thomas \& Chess, 1977; Zwickel, 2009). Therefore, it is assumed that

287 temperamental characteristics remain relatively stable within and across the first years

288 of life (Bornstein et al., 2019; Carnicero et al., 2000 Pedlow et al., 1993 Peters-Martin

289 \& Wachs, 1984; Rothbart et al., 2000; Rubin et al., 2002). Therefore, unlike the items of

290 the other scales, the items in the social-emotional scale are only asked at one point in

291 time per scale and do not follow the AoA approach. Because ToM is often considered as

292 not being stable, in the next version of the APP, repeated presentations of particular

293 questionnaires will be implemented.

294 To assess the children's social-emotional development, we included four scales

295 measuring attention, early temperament, and social-cognitive development between the

296 ages of 3 months and 6 years: 1) The Infant Behavior Questionnaire for infants aged 3

297 to 12 months (IBQ-R, Gartstein \& Rothbart, 2003). 2) The Early Childhood Behavior

298 Questionnaire (ECBQ, Putnam et al., 2006) for children between 18 and 36 months

299 (Putnam et al., 2006). 3) The Children's Behavior Questionnaire (CBQ) for children 3 
300 years and older (Rothbart et al., 2001) that is suitable for the age range between 3 and

3017 years. 4) The Children's Social Understanding Scale (CSUS, Tahiroglu et al., 2014) to

302 assess children's ToM. For details on the measures and answer options see Appendix

303 For detailed information about the validity, and the reliability, we refer to the original

304 publications mentioned.

\section{Specifications of the APP}

306 In the following, we first provide information about data security, storage, and

307 ethical approval, followed by technical details about the programming structure and set 308 of the APP.

\section{Ethics and Data Security}

\section{Ethics Approval and Informed Consent}

311 The study protocol and the procedures were approved by the local ethics

312 committee (Reference Number 20.6.5) and are in accordance with the ethical standards

313 of the 1964 Helsinki declaration and its later amendments. Caregivers are, for example,

314 free to stop using the APP at any time without giving reasons for justification. All

315 caregivers who intend to use the APP provide informed consent. No incentive other

316 than the free use of the APP is provided to the children and their caregivers by the

317 research unit Developmental Psychology at the Department of Psychology and the

318 Jacobs Center for Productive Youth Development of the University of Zurich

319 (henceforth referred to as HOST). When registering for the APP, a user explicitly agrees

320 to the data processing as set out in the Terms of Use for the APP and the Privacy

321 Policy by the University of Zurich (UZH). The HOST will continuously refine the APP.

322 At some instances, this will lead to changes in the data processing by the HOST. Users

323 will be notified of such changes in an appropriate manner (e.g., at the next login).

\section{Data Security and Data Protection}

After installation, declarations of consent under data protection law are obtained

326 and a declaration is made as to which data are shared with the research unit 
327 "Developmental Psychology" at the UZH and which is stored locally on the device but

328 not forwarded to the server. All non-local data are sent via authentication tokens to a

329 virtual server hosted and maintained by the IT Services of the UZH. Only UZH staff

330 responsible for the maintenance of the server, the programmers for update functions,

331 and authorised staff of the Department of Psychology and the Jacobs Center for

332 Productive Youth Development at the UZH have access to the data. The data security

333 strategy has been approved by the Data Security Office of the UZH and the Data

334 Security Office of the Canton of Zurich / Switzerland

335 (https://www.zh.ch/de/politik-staat/datenschutz.html). The data protection

336 declaration can be viewed under https://t.uzh.ch/1dA. Cooperating research units can

337 be granted access to parts of the data if they sign a data delivery contract with the

338 HOST and when they have received a declaration of consent from the participating

339 caregivers. All information about data protection is available on https://osf.io/jxspz/.

\section{Data Shared with the Researchers}

341 Data transmitted to the HOST is restricted to the information related to the

342 questions asked (see https://osf.io/ar7xp/). Other data are collected solely within the

343 APP and not synchronised with the HOST. This includes the e-mail address of the user,

344 the name of the child, any photo or video material collected, any individual comments

345 on specific developmental steps, own entries for personal events. These data are stored

346 locally and encrypted on the caregivers' own mobile device. The HOST has no access to

347 these data.

\section{Technical Specifications}

\section{Operating Systems}

$350 \quad$ Front end and back end of the APP have been programmed and are maintained

351 by the companies Hybrid Heroes GmbH (Berlin, Germany,

352 http://www.hybridheroes.de) and Smartcode (Zürich, Switzerland, 


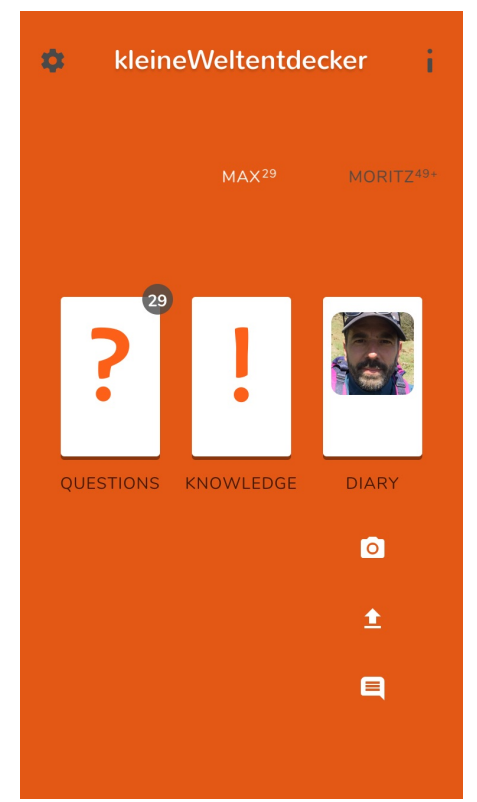

(a)

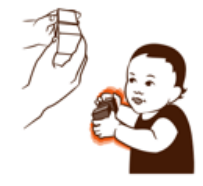

If you show Max how to bang two blocks together, does Max imitate this action?

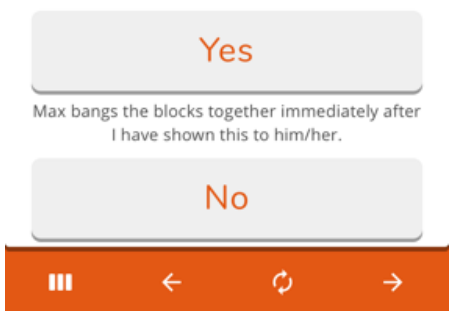

(b)

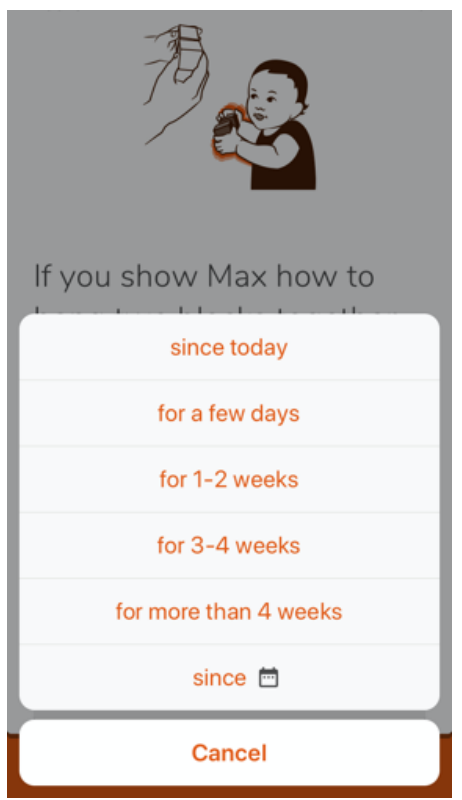

(c)

\section{Figure 1}

Depiction of the APP navigation: (a) Home screen of user navigation, (b) item and answer options, (c) options to indicate the time since when a child shows a particular skill.

http://www.smartcode.ch) as a hybrid app that works for the operation systems iOS

354 and Android.

\section{Graphical User Interface (GUI)}

The home screen of the APP includes the following sections (see Figure 1):

357 1) Settings: Here, basic settings can be adjusted such as the frequency and time of push

358 notifications to inform caregivers that new questions are available for the APP,

359 username and password, and whether or not the development of one's own child shall be

360 compared with the available norm values. 2) Questions: Caregivers are provided with

361 the specific questions/ items about developing skills in the four domains. All items and

362 milestones are illustrated with pictures. The visual appearance of the items is based on

363 a stack of cards. Each card contains a question about a particular skill on the front

364 side. Swiping the card to the left reveals the next card and item. A swiping movement

365 to the right brings back the previous item. Each card can be flipped over to reveal 
section 3) Knowledge on the back side that includes information about the skill at question and its typical development. 4) Diary: For the cognition and the motor scales,

368 caregivers see the acquired skills of their children with the corresponding date of 369 attainment 2 Caregivers who indicated in the Settings that they wished to compare

370 their child's data with the available norm data can access this norm distribution derived

371 from the whole population of children included in the APP. In the Diary section,

372 caregivers can also add individual personal events that are not included in the set of 373 questions (e.g., the appearance of the first tooth, the first day at the nursery, birthdays,

374 etc). In this section, caregivers can furthermore upload pictures to enrich their diary.

375 These individual personal events and pictures will not be shared with the HOST (see

376 Ethics and Data Security. 5) Further options: Further pages contain core data of the

377 children (date of birth, sex).

\section{Scientific Illustrations}

A scientific illustrator (Nadja Stadelmann, http://www.nadjastadelmann.ch)

380 created illustrations for all items to visualise the corresponding skills. These

381 illustrations visualise the domain (demographic information, socio-emotional skills) or a

382 concrete developmental skill. She developed illustrations for four children of both sexes

383 in different ages: at 4, 12, 24, and 48 months. Exemplary illustrations are shown in

384 Figure 2, The children are depicted using a planar style, the caregivers are depicted

385 using a linear style. This resulted in a strong focus on the child's behaviour. To

386 illustrate the movements, single movement steps are colour highlighted using hue

387 saturation lightness (see Figure $3(\mathrm{~b})$ ). In some illustrations, the order of steps was

388 accompanied with coloured arrows or numbers (see Figure 3 (c)). In the APP, the user

389 can assign one of eight colours to a child. The illustrations are using this basic colour in

390 combination with the hue saturation colour gradation.

\footnotetext{
2 The respective answer options only allowed a systematic depiction of the AoAs for these two scales
} 


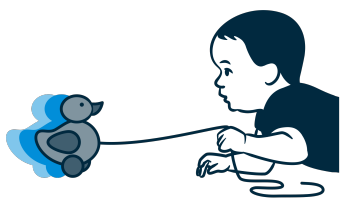

(a)

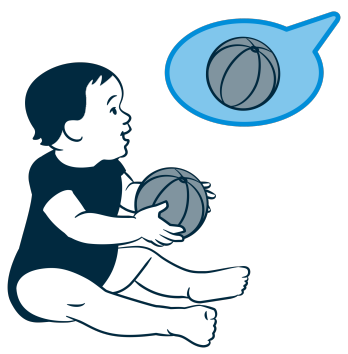

(b)

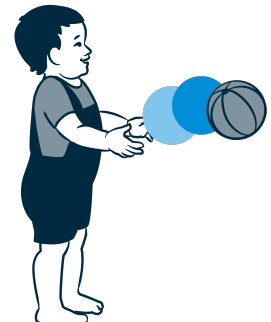

(c)

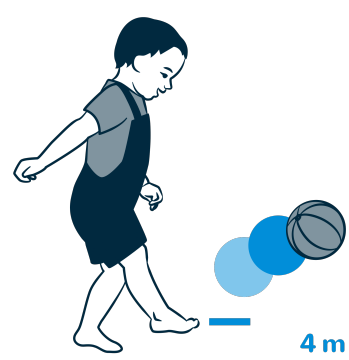

(d)

\section{Figure 2}

Depiction of the four age groups: (a) An infant at 4 months, (b) an infant at 12 months, (c) a toddler at 24 months, and (d) a preschooler at 48 months

\section{Answer Options}

393 the skill (Yes) or not (No). If caregivers indicate "Yes", they are further asked to

394 indicate since when the child mastered the skill. The following options are available:

395 "since today", "for a few days", "for 1-2 weeks", "for 3-4 weeks", "for more than 4

396 weeks", "since...(choose exact date)", see Figure 1. For some of the scales (e.g., the

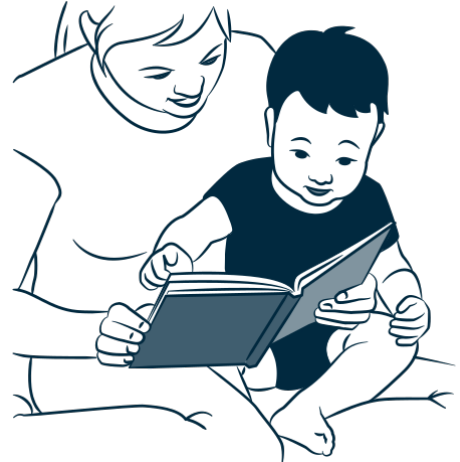

(a)

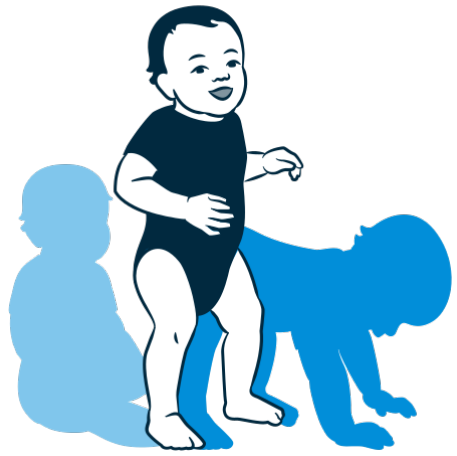

(b)

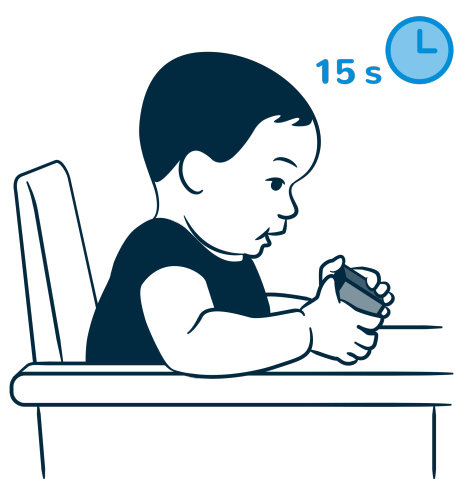

(c)

\section{Figure 3}

Exemplary Illustrations: (a) Child in planar style, adult in linear style, (b) movement of a child while moving from sitting to free standing, (c) additional information depicted by the duration in $s$. 
397 language scale, the social-emotional scale), the answer format deviated from this general

398 procedure, see Appendix for more information. For each item, the questions and the

399 answering options are presented in combination with information about the qualitative

400 criteria of the item and the represented skill. The wording depends on the respective

401 items. For the small "experiments" the items ask whether "my child does x"?. For other

402 skills, the items ask whether the child is in principle able to do x (e.g., "can stand on

403 one leg") because the child does not always stand on one leg but might have shown this

404 behaviour already.

\section{Procedure}

\section{Caregiver Information to Milestones}

407 For all items in the final APP scale, we created informative content about the

408 skills. We summarised precursors and the development around the milestone and

409 provided examples and contextual information. Furthermore, advice and inputs are

410 given on how to foster developmental progress and which training would best fit this

411 developmental phase.

\section{Languages}

413 The APP is currently available in four different languages (German, French,

414 Italian, British English). This includes three of the four languages spoken in

415 Switzerland (except Rhaeto-Romance). Caregivers can choose in which language to use

416 the APP. The range of languages can be expanded at any time, researchers around the

417 globe are welcome to contact the authors.

\section{Prompts and Repetition of Items}

After installation, caregivers are prompted via push notifications periodically in

420 time intervals between "once a week" and "once a month" to answer a short set of items

421 about their children's development. The time interval can be selected in the Settings

422 section (see Graphical User Interface (GUI) . It is possible to answer items at any time.

423 If the caregivers respond with "No" to a certain item, this question will be repeated 
424 after a period of two weeks. The APP currently selects the items based on the earliest

425 possible time (age in days) at which this skill was shown in a child from the data

426 coming from the norm sample within the app. With this approach, children who have a

427 comparably early AoA are not missed. To not miss children who have later AoAs, the

428 questions are repeated until the caregiver indicate that the skill has been observed.

\section{Psychometric Properties of Scale}

In the following, information about the psychometric properties of the APP

431 scales is provided including objectivity, reliability, construct validity, and criterion

432 validity. We report the psychometric properties for the cognition, language, and motor

433 scale (except for the pragmatic language skills assessed by the Orion's Pragmatic

434 Language Skills Questionnaire, Ghahari et al., 2017). The psychometric properties of

435 the social-emotional scales are well-documented in the respective publications

436 mentioned above.

\section{Participants}

438 In the sample used to assess the psychometric properties, we included all APP

439 data points provided by the caregivers until the date of data extraction (11 March

440 2020). The data were filtered for outliers and test users using the following exclusion

441 criteria: 1) children were older than 6 years, 2) caregivers were younger than 20 years or

442 older than 55 years 3 , 3) caregivers provided a highly unlikely birth country (e.g.,

443 Antarctica), 4) caregivers answered fewer than 10 questions, 5) the AoA of a skill was

444 before the birth of the respective child 4 . The original sample consisted of 5067 children.

445 The application of the filtering criteria resulted in a final validation sample of 2385

446 children (1112 girls, 1265 boys, and 8 children for whom caregivers chose 'other' as

447 indication of sex). The mean age of the children at the date of data extraction was

$448 M_{\text {children }}=791$ days, $S E_{\text {children }}=11$ days. In this validation sample, the APP was used

\footnotetext{
${ }^{3}$ Because there were only very few caregivers outside of this age window we categorised them as test users.

${ }^{4}$ In the latest version of the APP, it is no longer possible to indicate an impossible AoA.
} 
449 by 1984 mothers, 294 fathers and 16 other caregivers, 91 did not answer this question.

450 The mean age of the APP user at the date of extraction was $M_{\text {user }}=36$ years,

$451 S E_{\text {user }}=0.08$ years.

452 For construct validation, we invited caregivers $(N=256)$ who filled out the

453 'pre'-questionnaires (see Participants: Target Group for their children to participate in

454 a lab study with their children. We compared caregivers' answers in the

455 'pre'-questionnaire to their child's performance in lab-based standardised tests. The

456 validation sample for the cognitive scale included 74 children $\left(n_{\text {girls }}=36, n_{\text {boys }}=38\right.$,

$457 M_{\text {age }}=734$ days, $S E=42$ days). validation sample for the motor scale included

45897 children $\left(n_{\text {girls }}=46, n_{\text {boys }}=51, M_{a g e}=873\right.$ days, $S E=63$ days $)$. The validation

459 sample for the language scale included 85 children $\left(n_{\text {girls }}=38, n_{\text {boys }}=47\right.$,

$460 M_{a g e}=1480$ days, $S E=60$ days $)$.

\section{Analyses Plan}

In the following sections, we describe the different psychometric properties of the

463 APP scales. To analyse objectivity and criterion validity, we used different multi-level

464 logistic regressions predicting either the AoA for the motor and cognitive items or the

465 language scale index for language skills by domain (motor or cognition), caregiver

466 education (mother and father), caregiver age (mother and father), app user (mother or

467 father), sex of the child, and pregnancy week in which the child was born (see also

468 Equation 1 in the Appendix. Details about the specific analyses are reported in the

469 respective sections below. To measure construct validity, we predicted children's

470 performance in lab-based tests with the answers of caregivers for the according items in

471 the APP assessed via the 'pre'-questionnaires using multi-level regressions for the

472 motor, cognition and language scale. As a reliability measure, we assessed the internal

473 consistency by calculating Cronbach's $\alpha$ separately for the different scales and age

474 ranges. 


\section{Table 2}

Psychometric Values for the Assessment of the Objectivity and Criterion Validity for the Motor and Cognition Items: Type III Analysis of Variance Table with Satterthwaite's Method.

\begin{tabular}{lrrrrrrr}
\hline & Sum Sq & Mean Sq & NumDF & DenDF & F value & $\operatorname{Pr}(>$ F $)$ & Sig. Level \\
\hline Pregnancy & 20943 & 20943 & 1 & 1700.2 & 7.118 & .008 & $* *$ \\
Sex & 517 & 259 & 2 & 1697.2 & 0.088 & .916 & \\
Age $_{\text {Mother }}$ & 206250 & 206250 & 1 & 1699.9 & 70.100 & $<.001$ & $* * *$ \\
Age $_{\text {Father }}$ & 20175 & 20175 & 1 & 1697.5 & 6.857 & .009 & $* *$ \\
Education $_{\text {Mother }}$ & 15525 & 3105 & 5 & 1696.8 & 1.055 & .384 & \\
Education $_{\text {Father }}$ & 14553 & 2911 & 5 & 1693.0 & 0.989 & .423 & \\
APP $_{\text {User }}$ & 4803 & 2401 & 2 & 1697.1 & 0.816 & .442 & \\
Domain & 103 & 103 & 1 & 206.0 & 0.035 & .852 & \\
\hline
\end{tabular}

Because there were only few cognitive items, we merged items of the motor and the cognitive items in this model and included domain as a factor. There was no effect of domain. The model accounted for $98.28 \%$ of the variance.

\section{Objectivity}

476 To assess objectivity, we analysed the influence of the APP users in our

477 regression on the AoA in the motor, cognitive, and language scales. That is, we tested

478 whether it made a difference whether the data were entered by mothers, fathers, or 479 other users. The results showed that the factor APP user had no influence on the 480 indicated AoA, see Tables 2 and 3.

\section{Reliability}

$482 \quad$ For all scales, we assessed the internal consistency by calculating Cronbach's $\alpha$ 483 separately for the scales and age ranges. See Table A1 for an overview of the results in 484 the single scales and age ranges. The results indicate a range between acceptable $485(\alpha>.70)$ and excellent $(\alpha>.90)$ reliabilities for almost all age ranges for the domains 486 of fine motor, gross motor, and language. Only the value for fine motor skills between 


\section{Table 3}

Psychometric Values for the Assessment of Objectivity and Criterion Validity for the Language Items: Type III Analysis of Variance Table with Satterthwaite's Method.

\begin{tabular}{lrrrrrrr}
\hline & Sum Sq & Mean Sq & NumDF & DenDF & F value & $\operatorname{Pr}(>F)$ & Sig. Level \\
\hline Age $_{\text {Days }}$ & 472779 & 472779 & 1 & 289.29 & 582.325 & $<.001$ & $* * *$ \\
Pregnancy $_{\text {week }}$ & 1589 & 1589 & 1 & 387.45 & 1.957 & .163 & \\
Sex & 4751 & 4751 & 1 & 410.92 & 5.852 & .016 & $*$ \\
Age $_{\text {Mother }}$ & 95 & 95 & 1 & 321.50 & 0.118 & .732 \\
Age $_{\text {Father }}$ & 362 & 362 & 1 & 315.64 & 0.446 & .505 & \\
Education $_{\text {Mother }}$ & 2053 & 513 & 4 & 321.49 & 0.632 & .640 \\
Education $_{\text {Father }}$ & 1350 & 270 & 5 & 336.51 & 0.333 & .893 \\
APP $_{\text {User }}$ & 2392 & 1196 & 2 & 310.82 & 1.473 & .231 & \\
\hline
\end{tabular}

The model accounted for $65.24 \%$ of the variance.

48712 and 18 months was slightly below the acceptable value of $\alpha=.70$. The reliability 488 scores for the cognition items were less solid and mostly ranged below $\alpha=.60$ with the 489 exception of the age range between 3 and 6 months $(\alpha=81)$.

\section{Validity}

\section{Construct Validity}

492 Each scale was validated for different age groups. We used the pre-questionnaire 493 (see Participants: Target Group) to assess caregivers' answers to items of the APP and 494 compared them to children's behaviour in the corresponding items of existing scales (see 495 below for details on which scales were chosen) using logistic regressions. We followed 496 the procedures and scoring guidelines of the existing scales.

497 For the motor scale, we tested children's motor skills with the motor items of the 498 Bayley Scales for Infant and Toddler Development III (BSID-III, Bayley, 2005) up to 42 499 months and with the Peabody Developmental Motor Scales (PDMS, Rhonda Folio \& 500 Fewell, 2000) for children older than 42 months. For the cognitive scale, we used the 501 items of the cognitive scale of the BSID-III (Bayley, 2005). For the language scale, we 
502

503

504

505

506

507

508

509

510

511

512

513

514

515

516

517

518

520

521

522

523

524

525

526 significantly predicted the TSVK score,

527 estimate $=1.450, S E=0.469, z=3.092, p=.003$, and the delay

528 estimate $=-0.065, S E=0.021, z=-2.999, p=.004$. The model accounted for $27.05 \%$

529 of the variance. Similarly, the grammar scale significantly predicted the PPVT score, 530 estimate $=18.321, S E=7.451, z=2.459, p=.024$. However, we did find no effect of 
531 delay, estimate $=-0.496, S E=0.546, z=-0.907, p=.376$. The model accounted for

$53241.42 \%$ of the variance. In sum, the standardised lab test performances were predicted

533 by caregivers' answers to the language scale questions of the APP, which shows

534 excellent content and predictive validity.

\section{Criterion Validity}

We investigated criterion validity by predicting the AoA outcomes and language

537 scores with factors that typically effect development. Here, we tested the pregnancy

538 week a child was born, child's sex, age and education of father and mother, and the

539 APP user (i.e., whether father, mother, or another caregiver provided the data) and

540 entered them into the model. The results are shown in Table 2 (Motor Scale and

541 Cognitive Scale), and Table 3 (Language Scale). For the motor and the cognition scale,

542 we found that AoA was predicted by the pregnancy week with births in earlier

543 pregnancy weeks being associated with later AoA, and effects of caregiver age, with

544 older caregivers showing later AoA, an effect that was stronger for mothers' age than

545 fathers' age. We found no effects for domain (cognition, motor), child's sex, caregiver

546 education, and APP user. For the language scale, we found that boys were evaluated as

547 having poorer language skills than girls and language skills increased with age, see

548 Table 3. In sum, factors that typically effect development such as pregnancy week or

549 gender also influenced the scores that were obtained by the caregivers. We therefore

550 conclude that the APP has sufficient construct validity.

551 Our analyses of the psychometric properties of the APP indicate a sufficient

552 objectivity, reliability, and validity for the motor and language scales. For the cognition

553 scale, reliability and validity measures need to be improved in future versions of the

554 APP by editing, including, or excluding individual items (see Discussion).

To further substantiate the validity of the items used and the general method of 557 ambulatory assessment based on a digital developmental diary, we present some initial

558 findings of what can be measured and whether and how previously reported findings are 
559 replicated. First, we present some data that describe the sample drawn for the current

560 purpose (see Descriptive Data and Demographics, date of data extraction: 11 March

561 2020). Second, we present a preliminary replication of the relationship of non-verbal

562 and verbal communication skills (see Communicative Skills, below).

\section{Descriptive Data and Demographics}

564 Caregiver answered on average 75 questions, ranging between 10 and 285. The

565 mean duration between registration and last usage (i.e., the mean length of usage) is

5664.32 months, ranging between 1 months and 16 months. On average, caregivers filled in

567 questions on 2.44 different days per month, ranging between 0.15 and 16 days.

568 Caregivers answered questions on average every 21 days $(M=21.43$ days, $S E=0.31)$.

569 Per day, caregivers answered on average 3.01 questions $(\mathrm{SE}=0.06)$ questions in

570 cognitive development, 12.53 questions $(S E=0.22)$ in motor development, 7.06

571 questions $(S E=0.25)$ in language, 2.89 questions $(S E=0.12)$ in social-emotional

572 development, 3.27 questions on physical measures and 10.7 questions $(S E=0.1)$ on

573 background variables.

574 Further, as an example of geographical distribution, we collected data on the

575 countries of birth and living, see Table A2. Currently, most of the users live in

576 Switzerland (54\%) and Germany (38\%), in total 88 countries of residence were

577 indicated. Finally, as one example for demographic variables, caregivers are asked to

578 indicate how their child was born, either via natural birth or via Caesarean section. The

579 Swiss Federal Statistical Office (Statistik, 2020) reports for the year 2017 that almost

580 one third $(32.3 \%)$ of all newborns $(n=85.990)$ in Switzerland were be born by

581 Caesarean section. The data collected in our APP reveal the percentage of $32.4 \%$

$582(n=790$ out of $N=2437)$. The two percentages are almost identical, with no statistical

583 difference between them, $\chi^{2}=0.009, p=.931$. While we don't claim that the data

584 generated by the unsupervised APP use are representative for the population, it seems

585 that they approximate population statistics in such key variables. 


\section{Communicative Skills}

Finally, we present data on a developmental psychological aspect of the APP

588 data: The development of the interrelation between non-verbal and verbal

589 communication. Previous research in laboratory settings reported a longitudinal

590 relation between joint attention and language development (Farrant \& Zubrick, 2012,

591 Morales et al., 2005). Children who had low levels of joint attention during infancy were

592 significantly more likely to have poor receptive vocabulary around age of 5 (Farrant \&

593 Zubrick, 2012). Also, children with lower scores in pointing at the age of 12 months

594 (pointing only with open hand but not yet with index finger) were at risk for language

595 delay one year later (Lüke et al., 2017). The analysis of a subset of infants $(n=198$,

$\left.596 n_{\text {girls }}=97, n_{\text {boys }}=101\right)$ taken from the APP data indicated that the onset of early joint

597 attention ability (i.e., child is looking from an object to caregivers and back)

598 significantly predicted the age at which infants spoke their first words,

$599 \beta=0.51, p<.001, F(3,194)=50.28, p<.001, R^{2}=0.44$. There was no main effect of

600 infants' sex, $\beta=-21.81, p=.563$, nor an interaction of sex and early joint attention,

$601 \beta=0.06, p=.495$. Infants who showed joint attention earlier in life also spoke sooner

602 (see Figure 4). This is in line with the above mentioned previous findings that infants'

603 developing non-verbal social-cognitive skills are longitudinally related to their emerging

604 language skills.

\section{Discussion}

In this paper, we present a new tool for the assessment of children's development

607 from birth to the age of 6 years. Via the use of a smartphone-based developmental

608 diary application (the kleineWeltentdecker App, referred to as the APP), caregivers can

609 track the emergence and the development of their children's skills in four major

610 developmental domains. The empirical validations of the reliability of the procedures

611 with children of different age groups have (except for the cognition items) confirmed the

612 robustness of the different measures implemented in the APP. In the following, we

613 discuss the psychometric properties, the goals, and the challenges of the APP. 


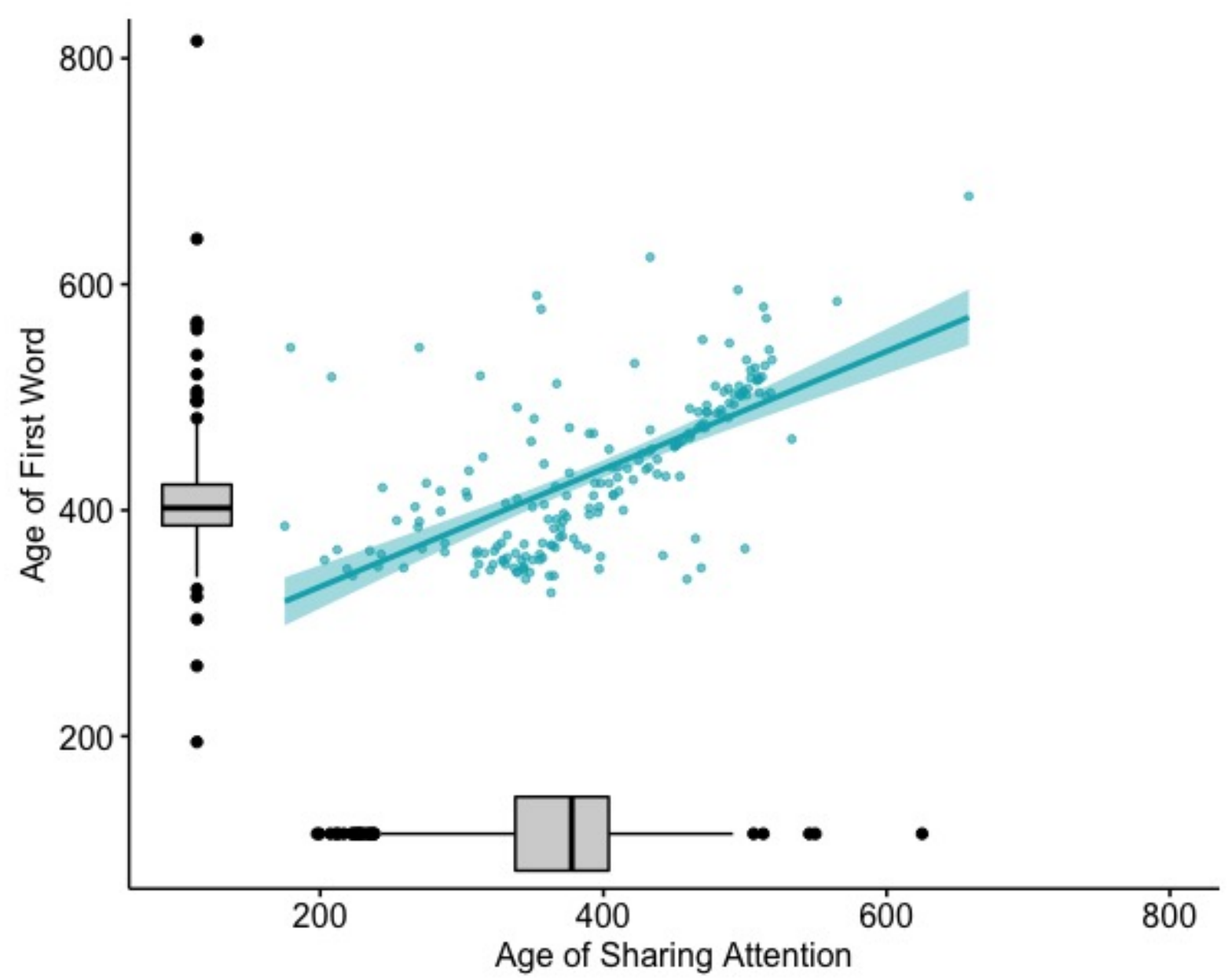

\section{Figure 4}

Relationship between AoA of Sharing Attention as an indicator of early joint attention ability and the AoA of First Word as an indicator of early language skills. Individual data points (blue) and box plots are illustrated.

\section{Psychometric Properties}

615 The assessment of the psychometric properties resulted in an overall positive 616 outcome. The high objectivity of the data is indicated by the fact that no differences 617 were found between the AoAs and the usage frequency between mothers, fathers, and 618 other caregivers, which was the case in all scales.

\section{Reliability}

620 The assessment of reliability resulted in mixed findings and the reliability

621 critically depended on the scale tested. Reliability was excellent for the language scale 
622 and good for the motor scale. There is room for improvement with respect to the

623 cognition scale, for which the reliability was generally below acceptability.

624 There several potential reasons that can explain the non-optimal results for the

625 cognition scale. First, the scale consists of fewer items $(n=34)$ compared to the other

626 scales (all $n \mathrm{~s}>100$ ), which might result in a larger variability of the results. Second, the

627 range of tested skills is relatively broad and thus heterogeneous. It ranges from simple

628 sensori-motor items to more complex tasks on memory or problem-solving. Third, and

629 probably most important, the items often involve the instructions for caregivers to

630 conduct a little "experiment" with their children. For example, basic memory functions

631 are assessed via the following item: "Try this little experiment: Put 3 pairs of shuffled

632 memory cards picture-side up on a table in two rows. Ask [Childname] to remember

633 where each card is. Then, turn over the cards one by one so that the pictures are no

634 longer visible. Now ask [Childname] where the paired pictures are, one by one." (Item

635 CG32). While the instructions are formulated as easy-to-understand, caregiver-friendly,

636 and unambiguous as possible, there is still room for variation in how exactly caregivers

637 perform these experiments and how the child's behaviour is interpreted. Also, the

638 relatively high cost for the caregiver to perform the task (e.g., getting up and searching

639 for memory cards) might have prompted a positive response even though the skill had

640 not yet been developed. Other instruments that assess children's cognitive development

641 require trained examiners to perform the assessment. Findings from citizen science

642 research are helpful to shed more light on this increase of variability: On the one hand,

643 citizen scientists can perform collections of valid basic data even when given only a brief

644 training (Darwall \& Dulvy, 1996, C. Evans et al., 2005; Fore et al., 2001; Graham et al.,

645 1996). On the other hand, data validity is decreased when citizen scientists are

646 confronted with more complex questions and observation tasks, such as observations in

647 astronomy (Balcom, 2015). In general, without proper training in experimental

648 protocols, citizen scientists (such as the caregivers who use the APP can be compared

649 to) are more likely to introduce variability into their data (Danielsen et al., 2005

650 M. Eaton et al., 2002). Applied to the present set of cognitive items, it might be the 
651 case that they are in general more difficult to evaluate than the motor or language

652 items. To conclude, the data collected with the current cognition items are not yet as

653 reliable as the other scales. Further developments are required to improve this scale.

\section{Validity}

Our analyses on construct and criterion validity yielded no effects of caregiver

656 education on the data. This indicates that caregivers of all educational backgrounds

657 respond similarly to the questions. Caregiver education is only a rough estimate for a

658 more global assessment of caregivers' socio-economic status (SES). We will, therefore,

659 evaluate whether a more differentiated assessment of SES (e.g., asking for income and

660 other aspects) is required and likewise feasible and accepted by caregivers. The present

661 data showed that children's sex had a significant effect on their language development.

662 Girls generally had earlier language AoAs than boys. This finding is well-established in

663 the field: Girls produce sounds and use words at an earlier age, have larger vocabularies,

664 greater grammatical complexity, and read sooner than boys (Bornstein et al., 2004;

665 Lange et al., 2016, Lisi et al., 2002, D. I. Miller \& Halpern, 2014 Reilly et al., 2019

666 Stolt et al., 2008). Interestingly, the AoA was related to the caregiver age. Children of

667 older caregivers had later AoAs. Previous research reports that caregiver income related

668 to better problem solving and language scores (Yeung et al., 2002) and that caregiver

669 job loss had an impact on their children's performance in school (e.g., Rege et al., 2011;

670 Stevens \& Schaller, 2011), an effect that seems already visible even before children enter

671 school (Mari \& Keizer, 2020). Caregiver income and their SES increase with caregiver

672 age (e.g., Featherman et al., 1988; Mclanahan, 2004 Powell et al., 2006; Ross \&

673 Mirowsky, 1999). Children from older caregivers should therefore have earlier AoAs

674 than children from younger caregivers. In light of this tendency, the present data are in

675 contrast with this previous data. However, the analysis of the demographics indicated

676 that the general level of education of the caregivers using the APP was relatively high

677 and variability was relatively low. Previous research shows that caregiver education and

678 children's outcome are considered at a bivariate level only, the relationship can be

679 curvilinear and disadvantageous for children with comparatively young or old caregivers 
680 (e.g., Powell et al., 2006). However, when considering additional factors such as SES or

681 family structure, the pattern typically becomes linear and caregiver age becomes

682 positively linked to child outcomes. These aspects require further attention as the

683 amount and the quality of the data increase. In general, the results of the analysis of

684 the psychometric properties are promising. This is particularly the case for the motor

685 and language scale whereas the results for the cognition scale are more heterogeneous.

\section{Goals and Challenges}

In the introduction, we formulated three major goals. We aim 1) to establish a

688 comprehensive data set of child development, 2) to have tool that accounts for the

689 variability on development across cultures beyond WEIRD countries, and 3) to

690 outsource data collection to caregivers. In the following, we discuss how the APP can

691 help researchers to reach these goals and the challenges that have yet to be met.

\section{Goal 1: Establish a Comprehensive Data Set of Child Development From}

\section{Age 0 to 6, Within and Across Individuals}

694 With the APP, we aim to obtain data that inform about the variability of

695 behaviour and its development in relation to contextual aspects. The APP measures

696 children's competencies in the cognitive, language, motor, and social-emotional domains

697 of development. Furthermore, questions on children's culture, SES, and language

698 background offer information on their environment. The analysis of data acquired by

699 the APP is not limited by time-consuming processes of manual coding of behavioural

700 data. For the analysis, it does not make a big difference whether the data set includes

70130 or 30.000 participants. The data of the APP will provide information that goes far

702 beyond what has been called the "taking snapshots of developmental outcomes"

703 approach (Adolph et al., 2008, Caspi et al., 1996), and has the potential to substantially

704 increase our understanding of developmental processes. The APP uses an

705 Age-of-Attainment (AoA) approach (W. O. Eaton et al., 2014) that is centred on the

706 date of emergence of a developmental skill. Individuals differ in their AoAs. This

707 particular variability is of key interest because it provides evidence about how long it 
708 takes individuals to reach a particular skill and to move to the next skill. That is, it

709 allows evaluating individual differences in the chronological AoA and the temporal

710 distances between the AoA of two (or more) different skills. Eventually, this allows a

711 detailed description of individual developmental trajectories and the identification of

712 the interrelations between skills within and across domains. These descriptions of

713 developmental trajectories are essential for the advancement of theories about children's

714 development and the acquired data will help to significantly increase our understanding

715 of developmental change in childhood.

\section{Goal 2: Account for the Variability on Development Across Cultures}

717 Previous research in developmental psychology has to a large extent been based

718 on WEIRD populations, which has recently been criticised (e.g., Nielsen et al., 2017).

719 Therefore, variability of behaviour and development is underestimated. The approach of

720 the APP allows moving beyond sampling from highly homogeneous (often WEIRD)

721 populations to a sample of large variability with respect to cultures and social contexts.

722 Data collected with the APP allows comparing development within and between

723 cultures and drawing conclusions from highly diverse samples. This approach helps to

724 fulfil the plea raised by, for example, Nielsen and colleagues (2017) that a "complete

725 understanding of the ontogeny and phylogeny of the developing human mind depends

726 on sampling diversity" (p.32), which receives further and increasing support by

727 numerous other researchers (Clegg \& Legare, 2016, Henrich et al., 2010c; Legare \&

728 Harris, 2016; Nielsen \& Haun, 2016; van Schaik \& Burkart, 2011). This sort of data as

729 measured by the APP is essential to broaden our theoretical understanding about which

730 aspects of the development of skills and traits are universal and which culture-specific.

\section{Goal 3: Outsourcing of Data Collection}

732 With the APP, we outsource data collection of longitudinal high-density data to

733 caregivers. With this, we aim to reduce the enormous personal and financial resources

734 associated with such data collection. Moving data collection from the controlled setting

735 of a laboratory to the "real, noisy world" and from the hands of trained and 
experienced experimenters to the caregivers comes with several challenges. Previous

737

738

745 satisfactorily.

\section{Challenge 1: Infrequent Use of the APP}

Research designs that use online surveys and smartphone applications are

748 attractive. They come at relative low cost and offer great flexibility (Barrios et al.,

749 2011; J. R. Evans \& Mathur, 2005; Fan \& Yan, 2010; Fricker \& Schonlau, 2002;

750 Kaplowitz et al., 2004 LaRose \& Tsai, 2014). At the same time, they are subject to

751 lower completion rates than conventional survey methods (Börkan, 2010; Jones \& Pitt,

752 1999; Manfreda et al., 2008; Sax et al., 2003, Shih \& Fan, 2008). It is therefore likely

753 that a substantial number of caregivers will not use the APP regularly. This will result

754 in a large amount of missing data. There may be ways that help to increase caregivers'

755 commitment. Incentives such as monetary compensation (Frick et al., 1999), loyalty

756 points (Göritz, 2008), or sweepstakes offering of a certain monetary value (LaRose \&

757 Tsai, 2014) have been shown to increase commitment in online studies (as indicated by

758 an increase in response rate to invitations and completion rate, but see Göritz (2006)

759 for null results). For an extensive overview of psychological and data collection via

760 internet, the reader is referred to the extant literature (e.g., Birnbaum, 2004 Manfreda

761 et al., 2008 Reips, 2002; Shih \& Fan, 2008). However, given the already large number

762 of participating caregivers ( $>4.000$; March 2020), it is not feasible to offer any form of

763 monetary incentive to all users. 
One potential option is to offer caregivers to participate in a lottery that takes

765 place periodically where caregivers can win a voucher that can be used worldwide (e.g.,

766 in online music or book stores). Lotteries seem to have a positive impact on participant

767 commitment (LaRose \& Tsai, 2014). Participation in the lottery could be automatised

768 or applied as an incentive if caregivers contributed a predetermined number of data

769 points within a given period. A second option is to implement the APP as a

770 supplementary measure in a more controlled setting of an existing ongoing longitudinal

771 study. In such a setting, a smaller number of caregivers who agreed to take part in a

772 study can be motivated via incentives more easily and reminded repeatedly to answer

773 the questions. With this approach, the usage and data of "unsupervised" caregivers can

774 be compared to a highly controlled sample of caregivers, which will provide further

775 insights to the interrelation between use and data quality. Two examples for this second

776 option are first, the study Children and digital media (KiDiM; "Kinder und Digitale

777 Medien", 2021) by the Marie Meierhofer Institute in Zurich, Switzerland. Here, APP

778 data complements the collection of longitudinal data on children's media use. Second,

779 in a planned study on the relation between nutrition and cognitive development,

780 currently prepared by by the USZ Neonatology section (LEARN; Natalucci et al.,

781 2021), APP data will be used as a continuous measure of the children's development

782 between the specified measurement times. One major aim for the future will be to use

783 the data coming from "supervised" and "unsupervised" users to identify different user

784 behaviour, its impact on the data quality, and ways to impute missing data.

785 High-resolution data from "supervised" users will thereby serve as a basis to impute

786 missing data of the low-resolution data of the "unsupervised" users.

\section{Challenge 2: General Reliability of Questionnaire Data}

788 One might in general be sceptical about the reliability of the data caregivers

789 report. They might be inclined to answer the questions about the development of their

790 children too optimistically for reasons of social desirability. Previous research on the

791 reliability between laboratory assessments and caregiver reports revealed inconsistent

792 effects. Whereas caregiver scores often correlate with professional assessment they 
793 likewise tend to be a poor predictor of infants at risk of developmental delay (e.g.,

794 Emond et al., 2005). Caregiver report and experimenter home visit observations seem

795 to converge when assessing the development of motor skills. For example, the Parent

796 Milestone Report Form has been shown to be a reliable and valid instrument to assess

797 infants' development of a number of gross motor skills via caregiver report (Adolph

798 et al., 2008, Bodnarchuk \& Eaton, 2004). Similarly, Miller and colleagues (2017) showed

799 no differences in the evaluation of receptive, expressive language, and fine motor skills

800 between caregiver report and direct assessment via experts. Further, laboratory tests

801 and experimenter home visits often underestimate children's early linguistic abilities

802 (Bates, 1993) and adults are generally good in estimating skills. Self-reports, for

803 example in language proficiency evaluation, converge with objective measures in adults

804 (e.g., Marian et al., 2007). Furthermore, the current approach receives support by the

805 fact that caregivers are with their children in many more and highly variable situations

806 than a laboratory setting is able to establish. This makes it more likely that they

807 observe the skill that the APP asks for and that caregivers' assessment of their children

808 is close in time to the first occurrence of a skill. In contrast, when caregivers are asked

809 to assess the emergence of their children's first words (around the age of 12 months)

810 retrospectively, these estimates show only weak reliability that decreases with increasing

811 age of their children $(r=0.27$ at age 3 and $r=-0.11$ at age 5 , Majnemer \&

812 Rosenblatt, 1994). The analysis of the APP user objectivity was generally successful,

813 the results showed that the factor "APP user" had no influence on the AoA identified.

814 However, one issue needs to be discussed more critically. While the reliability was

815 evaluated by two independent observers, a caregiver via the questionnaire and a

816 researcher in the controlled laboratory environment, this was not the case in the

817 objectivity rating, which may have caused selection effects (e.g., fathers who are more

818 involved in raising their children might be more inclined using the APP and might,

819 therefore, be better observers). This is a limitation for larger conclusions concerning

820 objectivity. However, for those caregivers who have been using the APP so far, this

821 does not seem to be a major problem. 
In general, while caregivers may be susceptible to social desirability effects, they

823 are still likely the most reliable source to determine whether or not a skill is in their

824 child's repertoire (Sachse \& Suchodoletz, 2008). Caregivers are in the unique position to

825 observe and interact with their children across many different situations. This makes

826 them likely to report the particular moment when a skill was observed for the first time.

827 The everyday interaction of caregivers with their children is furthermore not subject to

828 issues with child motivation and cooperation and has been established as a valuable way

829 to quickly and cost-effectively add information important for the detection of

830 developmental delays (e.g., Nordahl-Hansen et al., 2014). These aspects result in an

831 increased application of caregiver reports for routine developmental screening that are

832 particularly helpful for the identification of children at risk for developmental delays, a

833 procedure that is in accordance with the recommendations by the American Academy

834 of Pediatrics (e.g., Emerson et al., 2016; Johnson \& Myers, 2007).

835 With respect to reliability, the current approach represents a trade-off between

836 feasibility and reliability. Optimally, caregivers would answer the question about the

837 emergence of a particular skill at several instances, repeated over several days to

838 account for the variability of the emergence of a particular skill (Adolph et al., 2008).

839 However, workload for the caregivers is already substantial in the current version of the

840 APP with up to 20 questions per notification. A further increase of this number will

841 result in a significant decrease of the number of participating caregivers. The current

842 approach relies on the fact that caregivers are required to answer the questions about

843 the development of their children in intervals of one week or one month. Considering

844 these circumstances, we are confident the APP a valuable and important tool that

845 avoids false negatives. Caregivers observe their children in a variety of different

846 situations and, triggered by the particular question posed by the APP, might bring

847 their children in situations in which the new skill is likely to be observed. It is

848 important to emphasise that the present scales will not replace any diagnosis of clinical

849 symptoms or developmental delays, which need an in-depth diagnosis of an expert

850 psychologist and/or paediatrician. 
Finally, to reliably assess the emergence of a developing skill, measuring daily

852 fluctuations would be optimal. The APP is not designed to fulfil this purpose but to

853 assess whether and when a child shows a skill with a defined quality. This approach is

854 inspired by developmental assessment tools like the Bailey Scales (Bayley, 2005) and by

855 the AoA approach (W. O. Eaton et al., 2014). The collected AoAs are snapshots,

856 subject to fluctuations over time. However, the questions are answered by the caregivers

857 not on the basis of a single observation at one point in time but on their everyday

858 observation of their children. The advantage of this approach is to collect data from a

859 large number of children in a relatively easy and convenient way and to be closer in

860 time to the actual AoA of a skill than traditional approaches.

\section{Challenge 3: Assessment of Language Skills}

862 While the APP may document children's development in the cognitive, motor,

863 and social-emotional domain, the measures on language development are not yet

864 comprehensively integrated. The current assessment of linguistic skills does not yet

865 include vocabulary. In an initial version of the APP, we included this aspect with almost

8662.000 items being asked to caregivers. The feedback caregivers provided indicated that

867 they became tired quickly of answering the vocabulary items due to the sheer number of

868 questions about single words their child might or might not speak at a given point in

869 time. For this reason, we removed the vocabulary section in the current version. One

870 potential approach to this challenge was recently introduced by Mayor and Mani (2019).

871 These authors presented a new methodological approach through which an estimation of

872 a child's vocabulary score (as assessed by the MacArthur Communicative Development

873 Inventories, CDI; Fenson et al., 2007) can be obtained by combining caregiver responses

874 on a limited set of words sampled randomly from the full CDI with the information

875 about how many children do or do not speak a particular word extracted from the

876 WordBank database (Frank et al., 2017). The findings show that using a reduced list of

877 only 25 words provided an accurate estimate of a child's vocabulary size for American

878 English, German, and Norwegian. Implementing an algorithm similar to the one used 
879 by Mayor and Mani (2019) can be one way to improve the procedure by substantially

880 reducing the number of vocabulary items. This is a plan for future implementation.

881 To sum up the challenges, there are reasons that caregiver assessment will be

882 both more and less precise than laboratory assessment. Collecting more data, asking

883 feedback from participating caregivers, and using this information to adjust items

884 accordingly will help to increase reliability in the future. For example, with more data

885 analysed, a "real" effect may become visible and the influence of individual children on

886 the data becomes less substantial.

\section{Anticipated Outcome and Significance}

Given the ubiquity of smartphones worldwide, smartphone applications

889 increasingly serve as digital support devices. With the developmental diary application

890 presented here, we put a portable data acquisition tool in the pocket of caregivers. The

891 value of this approach is high (in times of the SARS-CoV-2 pandemic in which this

892 paper was partially written, even more so). It includes longitudinal data of a potentially

893 large-scale, population-based sample. The sample size is not restricted to areas or by

894 limited (financial and human) resources. This approach allows to move beyond

895 sampling from highly homogeneous (often WEIRD) populations to a sample of great

896 variability with respect to cultures and social contexts. The data collected with the

897 APP allow comparing development within and between cultures and drawing

898 conclusions from highly diverse samples. Eventually, this allows a detailed description

899 of individual developmental trajectories, and the identification of the interrelations

900 between skills within and across domains. With the approach presented here, data are

901 collected from more children in more places at a higher frequency than it is possible

902 with moderated testing, either in-person or online. The descriptions of developmental

903 trajectories derived from these data are essential for the advancement of theories about

904 children's development and the acquired data will help to increase our understanding of

905 developmental change in childhood. 


\section{Disclosures}

\section{Author Contributions}

908 M. M. Daum, K. Antognini, M. Beisert, M. Bleiker, A. Gampe, I. Kurthen,

909 L. Maffongelli, and S. Wermelinger jointly generated the idea for the APP. All authors

910 contributed to the development of the different scales, A. Gampe and S. Wermelinger

911 wrote the analysis code and analysed the data, M. M. Daum wrote the first draft of the

912 manuscript, and all authors critically edited it. All authors approved the final

913 submitted version of the manuscript.

\section{Conflict of Interest}

915 The authors declare that there were no conflicts of interest with respect to the 916 authorship or the publication of this article.

\section{Acknowledgments}

918 We express our deep gratitude to a large number of people who were

919 substantially involved in the development of the APP: Rico Leuthold

920 (http://www.smartcode.ch) for programming the backend of the APP and Jan Gerwin

921 and Christian Justus (http://www.hybridheroes.de) for programming the frontend,

922 Christof Täschler (http://www.christoftaeschler.ch) for the UI/UX design, and Nadja

923 Stadelmann (http://www.nadjastadelmann.ch) for the scientific illustrations. Andrea

924 Grössbauer, Eva Pouwer, and Robert Weniger for their support in data protection

925 matters, Nadia Steiner for her support with legal questions, Ingo Jörissen, Jacqueline

926 Martinelli, Stefan Mischke, and Frank Schleich for their support with the UZH servers,

927 and Wolfgang Henggeler and Patrick Sticher for the trademark registration. Ramona

928 Abrecht, Ingo Besserdich, Leonie Hartmann, and Christina Tschan inserted the

929 questions and answers and worked on the knowledge texts, Sonja Brunschweiler has

930 proofread them. Jonas Gähwiler, Maximilian Haas, Natascha Helbling, Mirella

931 Manfredi, Matthew Rockey, and Virginie Rusca for various translation work. Nicole

932 Besson, Alexandra Ritter, Rebekka Rüesch, Isabella Schwyzer, and Nicole Zahnd have 
933 validated the answers given by the caregivers by testing children in our lab. Julia

934 Brehm, Kerstin Clausen, Julia Fenkl, Ebru Ger, Sarah Hauser, Karin Hollermayer,

935 Liridona Hoti, Lara Keller, Lea Mörsdorf, Petra Moser, Fiona Pugin, Meret Roth,

936 Larissa Stuber, and Freya Zacher for testing the APP again and again and providing

937 useful inputs. Finally Lisa Wagner and Sabrina Beck for commenting on a previous

938 version of this manuscript. Without the help of all these people involved, this project

939 would not have been possible.

\section{Funding}

$941 \quad$ The project was supported by the Department of Psychology

942 (http://www.psychologie.uzh.ch) and the Jacobs Center for Productive Youth

943 Development (http://www.jacobscenter.uzh.ch) of the University of Zurich.

\section{Open Practices Statement: Data availability, materials, and online resources}

945 The items of all the scales and information about data protection are available

946 on https://osf.io/ar7xp/. The data sets generated during and/or analysed during the

947 current study are not publicly available due to the current data protection guidelines,

948 see section Data Security and Data Protection but are available from the corresponding

949 author on reasonable request.

\section{Ethical approval}

951 The study protocol and the procedures were approved by the local ethics

952 committee (Reference Number 20.6.5) and are in accordance with the ethical standards

953 of the 1964 Helsinki declaration and its later amendments. Caregivers are, for example,

954 free to stop using the APP at any time without giving reasons for justification. All

955 caregivers who intend to use the APP provide informed consent. No incentive other

956 than the free use of the APP is provided to the children and their caregivers by the

957 Research Unit Developmental Psychology at the Department of Psychology and the

958 Jacobs Center for Productive Youth Development of the University of Zurich (HOST).

959 When registering for the APP, a user explicitly agrees to the data processing as set out 
960 in the Terms of Use for the APP and the Privacy Policy by the University of Zurich.

961 The HOST will continuously refine the APP. At some instances, this will lead to

962 changes in the data processing by the HOST. Users will be notified of such changes in

963 an appropriate manner (e.g., at the next login). 
965 Adolph, K. E. \& Hoch, J. E. (2019). Motor development: Embodied, embedded, enculturated, and enabling. Annual Review of Psychology, 70(1), 141-164. https://doi.org/10.1146/annurev-psych-010418-102836

968 Adolph, K. E., Vereijken, B. \& Shrout, P. E. (2003). What changes in infant walking and why [_eprint: https://srcd.onlinelibrary.wiley.com/doi/pdf/10.1111/1467-8624.7402011]. Child Development, 74 (2), 475-497. https://doi.org/10.1111/1467-8624.7402011

972 Adolph, K. E., Young, J. W., Robinson, S. R. \& Gill-Alvarez, F. (2008). What is the shape of developmental change? Psychological Review, 115(3), 527-543. https://doi.org/10.1037/0033-295x.115.3.527

975 Aslin, R. N. (2007). What's in a look? Developmental Science, 10(1), 48-53. https://doi.org/10.1111/J.1467-7687.2007.00563.X

977 Balcom, B. (2015). Improving crowdsourcing and citizen science as a policy mechanism for NASA. New Space, 3(2), 98-116. https://doi.org/10.1089/space.2015.0017

979 Barrios, M., Villarroya, A., Borrego, Á. \& Ollé, C. (2011). Response rates and data quality in web and mail surveys administered to PhD holders. Social Science Computer Review, 29(2), 208-220. https://doi.org/10.1177/0894439310368031

982 Bates, E. (1993). Comprehension and production in early language development:

983 Comments on savage-rumbaugh et al. Monographs of the Society for Research in Child Development, 58(3), 222-242.

985 Bayley, N. (1993). Bayley scales of infant development (2nd). Psychological Corporation. 986 Bayley, N. (2005). Bayley scales of infant and toddler development (Third). Pearson Education, Inc.

988 Birnbaum, M. H. (2004). Human research and data collection via the internet. Annual Review of Psychology, 55(1), 803-832. https://doi.org/10.1146/annurev.psych.55.090902.141601

991 Bishop, D. V. M. (2003). The children's communication checklist version 2 (CCC-2) psychological corporation. Psychological Corporation. 
993 Bodnarchuk, J. L. \& Eaton, W. O. (2004). Can parent reports be trusted?: Validity of 994 daily checklists of gross motor milestone attainment. Journal of Applied 995 Developmental Psychology, 25(4), 481-490. https://doi.org/10.1016/j.appdev.2004.06.005

997 Börkan, B. (2010). The mode effect in mixed-mode surveys: Mail and web surveys.

$998 \quad$ Social Science Computer Review, 28(3), 371-380. https://doi.org/10.1177/0894439309350698

1000 Bornstein, M. H., Hahn, C.-S. \& Suwalsky, J. T. D. (2013). Physically developed and 1001 exploratory young infants contribute to their own long-term academic achievement. Psychological Science, 24(10), 1906-1917.

1004 Bornstein, M. H., Hahn, C.-S. \& Haynes, O. M. (2004). Specific and general language 1005 performance across early childhood: Stability and gender considerations [Publisher: SAGE Publications Ltd]. First Language, 24(3), 267-304. https://doi.org/10.1177/0142723704045681

1008 Bornstein, M. H., Hahn, C.-S., Putnick, D. L. \& Pearson, R. (2019). Stability of child 1009 temperament: Multiple moderation by child and mother characteristics. British $1010 \quad$ Journal of Developmental Psychology, 37(1), 51-67.

1011 https://doi.org/10.1111/bjdp.12253

1012 Bronfenbrenner, U. (1992). Ecological systems theory. Six theories of child development: 1013 Revised formulations and current issues (pp. 187-249). Jessica Kingsley $1014 \quad$ Publishers.

1015 Campbell, R. V. D. \& Weech, A. A. (1941). Measures which characterize the individual 1016 during the development of behavior in early life. Child Development, 12(3), 1017 217-236. https://doi.org/10.2307/1125721

1018 Campos, J. J., Anderson, D. I., Barbu-Roth, M. A., Hubbard, E. M., Hertenstein, M. J. 1019 \& Witherington, D. (2000). Travel broadens the mind. Infancy, 1(2), 149-219. 1020 https://doi.org/10.1207/S15327078IN0102_1 
1021 Carnicero, J. A. C., Pérez-López, J., Salinas, M. D. C. G. \& Martínez-Fuentes, M. T.

1022 (2000). A longitudinal study of temperament in infancy: Stability and

1023 convergence of measures. European Journal of Personality, 14(1), 21-37.

$1024 \quad$ https://doi.org/10.1002/(SICI)1099-0984(200001/02)14:1<21::AID-

$1025 \quad$ PER367>3.0.CO;2-A

1026 Cascales, T. (2011). Questionnaire sur le comportement du nourrisson - forme révisée.

1027 french translation of gartstein, 6 rothbart's, infant behavior questionnaire -

$1028 \quad$ revised.

1029 Caspi, A., Moffitt, T. E., Thornton, A., Freedman, D., Amell, J. W., Harrington, H.,

1030 Smeijers, J. \& Silva, P. A. (1996). The life history calendar: A research and

1031 clinical assessment method for collecting retrospective event-history data.

1032 International Journal of Methods in Psychiatric Research, 6(2), 101-114. https:

1033 //doi.org/10.1002/(SICI)1234-988X(199607)6:2<101::AID-MPR156>3.3.CO;2-E

1034 Clegg, J. M. \& Legare, C. H. (2016). A cross-cultural comparison of children's imitative

1035 flexibility. Developmental Psychology, 52(9), 1435-1444.

$1036 \quad$ https://doi.org/10.1037/dev0000131

1037 Cozzi, P., Putnam, S. P., Menesini, E., Gartstein, M. A., Aureli, T., Calussi, P. \& 1038 Montirosso, R. (2013). Studying cross-cultural differences in temperament in

$1039 \quad$ toddlerhood: United states of america (US) and italy. Infant Behavior and

1040 Development, 36(3), 480-483. https://doi.org/10.1016/j.infbeh.2013.03.014

1041 Danielsen, F., Burgess, N. D. \& Balmford, A. (2005). Monitoring matters: Examining

1042 the potential of locally-based approaches. Biodiversity $\mathscr{E}$ Conservation, 14(11),

1043 2507-2542. https://doi.org/10.1007/s10531-005-8375-0

1044 Darwall, W. R. T. \& Dulvy, N. K. (1996). An evaluation of the suitability of

1045 non-specialist volunteer researchers for coral reef fish surveys. mafia island,

1046 tanzania - a case study. Biological Conservation, 78(3), 223-231.

1047 https://doi.org/10.1016/0006-3207(95)00147-6

1048 Davis, B. E., Moon, R. Y., Sachs, H. C. \& Ottolini, M. C. (1998). Effects of sleep

1049 position on infant motor development. Pediatrics, 102(5), 1135-1140. 
1050 Dinehart, L. \& Manfra, L. (2013). Associations between low-income children's fine 1051 motor skills in preschool and academic performance in second grade. Early Education and Development, 24(2), 138-161. https://doi.org/10.1080/10409289.2011.636729

1054 Dunn, L. M. \& Dunn, D. M. (2007). PPVT-4 - peabody picture vocabulary test

1055 (4th ed.). Hogrefe, Verlag für Psychologie. Retrieved April 27, 2018, from https: //www.testzentrale.ch/shop/peabody-picture-vocabulary-test-4-ausgabe.html

1057 Eaton, M., Gregory, R. \& Farrar, A. (2002). Bird conservation and citizen science 1058 counting, caring and acting. Ecosystem, 23, 5-13.

1059 Eaton, W. O., Bodnarchuk, J. L. \& McKeen, N. A. (2014). Measuring developmental 1060 differences with an age-of-attainment method. SAGE Open, 4(2), 1061 2158244014529775. https://doi.org/10.1177/2158244014529775

1062 Emerson, N. D., Morrell, H. E. R. \& Neece, C. (2016). Predictors of age of diagnosis for 1063 1064 children with autism spectrum disorder: The role of a consistent source of

1066 Emond, A., Bell, J. C. \& Heron, J. (2005). Using parental questionnaires to identify medical care, race, and condition severity. Journal of Autism and Developmental developmental delay. Developmental Medicine and Child Neurology, 47(9),

1069 Evans, C., Abrams, E., Reitsma, R., Roux, K., Salmonsen, L. \& Marra, P. P. (2005). The neighborhood nestwatch program: Participant outcomes of a citizen-science

1073 Evans, J. R. \& Mathur, A. (2005). The value of online surveys [Publisher: Emerald 1074 Group Publishing Limited]. Internet Research, 15(2), 195-219. https://doi.org/10.1108/10662240510590360

1076 Fan, W. \& Yan, Z. (2010). Factors affecting response rates of the web survey: A systematic review. Computers in Human Behavior, 26 (2), 132-139. https://doi.org/10.1016/j.chb.2009.10.015 
1079 Farrant, B. M. \& Zubrick, S. R. (2012). Early vocabulary development: The importance 1080 of joint attention and parent-child book reading. First Language, 32(3), 343-364. https://doi.org/10.1177/0142723711422626

1082 Featherman, D. L., Spenner, K. I. \& Tsunematsu, N. (1988). Class and the socialization 1083 of children: Constancy, change, or irrelevance? Child development in life-span

1085 Fenson, L., Bates, E., Dale, P. S., Marchman, V. A., Reznick, J. S. \& Thal, D. J. 1086 (2007). MacArthur-bates communicative development inventories. Paul H. Brookes Publishing Company Baltimore, MD.

1088 Folio, M. R. \& Fewell, R. R. (2000). Peabody developmental motor scales, second edition 1089 (PDMS-2). PRO-ED, Inc.

1090 Fore, L. S., Paulsen, K. \& O'Laughlin, K. (2001). Assessing the performance of 1091 volunteers in monitoring streams. Freshwater Biology, 46(1), 109-123.

1093 Frank, M. C., Bergelson, E., Bergmann, C., Cristia, A., Floccia, C., Gervain, J., 1094 Hamlin, J. K., Hannon, E. E., Kline, M., Levelt, C., Lew-Williams, C., Nazzi, T., Panneton, R., Rabagliati, H., Soderstrom, M., Sullivan, J., Waxman, S. \& Yurovsky, D. (2017). A collaborative approach to infant research: Promoting

1099 Frick, A., Bächtiger, M.-T. \& Reips, U.-D. (1999). Financial incentives, personal information and drop-out rate in online studies. Dimensions of Internet science,

1102 Frick, A. \& Wang, S.-H. (2013). Mental spatial transformations in 14- and 16-month-dld infants: Effects of action and observational experience. Child development.

1105 Fricker, R. D. \& Schonlau, M. (2002). Advantages and disadvantages of internet research surveys: Evidence from the literature [Publisher: SAGE Publications Inc]. Field Methods, 14(4), 347-367. https://doi.org/10.1177/152582202237725 
1108 Gartstein, M. A. \& Rothbart, M. K. (2003). Studying infant temperament via the

1109

1110

1111 Ghahari, S., Hassani, H. \& Purmofrad, M. (2017). Pragmatic competency and

1124 Goupil, L. (n.d.). Questionnaire sur le comportement de la petite enfance. obsessive-compulsive disorder: A comparative assessment with normal controls. Journal of Psycholinguistic Research, 46(4), 863-875. https://doi.org/10.1007/s10936-016-9467-6

Goldsmith, H. H. \& Campos, J. J. (1982). Toward a theory of infant temperament. In R. N. Emde \& R. J. Harmon (Eds.), The development of attachment and affiliative systems (pp. 161-193). Springer US. Retrieved April 1, 2020, from https://doi.org/10.1007/978-1-4684-4076-8_13

Göritz, A. S. (2006). Cash lotteries as incentives in online panels. Social Science Computer Review, 24(4), 445-459. https://doi.org/10.1177/0894439305286127

Göritz, A. S. (2008). The long-term effect of material incentives on participation in online panels. Field Methods, 20(3), 211-225. https://doi.org/10.1177/1525822X08317069 https://research.bowdoin.edu/rothbart-temperament-questionnaires/instrumentdescriptions/the-childrens-behavior-questionnaire/

Graham, K., Collier, B., Bradstreet, M. \& Collins, B. (1996). Great blue heron (ardea herodias) populations in ontario: Data from and insights on the use of volunteers. Colonial Waterbirds, 19(1), 39-44. https://doi.org/10.2307/1521805

Gredebäck, G., Gottwald, J. M. \& Daum, M. M. (2021, March 26). How our hands shape our minds: Six developmental pathways [type: article]. PsyArXiv. https://doi.org/10.31234/osf.io/378rz

Green, E., Stroud, L., Bloomfield, S., Cronje, J., Foxcroft, C., Hunter, K. \& Venter, D. (2016). Griffiths scales of child development (3rd ed.) Hogrefe Ltd.

Grob, A., Reimann, G., Gut, J. \& Frischknecht-Brunner, M.-C. (2013). IDS-p intelligence and development scales - preschool - hogrefe verlag. Hogrefe Verlag. 
1137 Hamaker, E. L. (2012). Why researchers should think "within-person": A paradigmatic rationale. In M. R. Mehl \& T. S. Connor (Eds.), Handbook of research methods for studying daily life (pp. 43-61). Guilford.

1140 Henrich, J., Heine, S. J. \& Norenzayan, A. (2010a). Beyond WEIRD: Towards a broad-based behavioral science. Behavioral and Brain Sciences, 33(2), 111. https://doi.org/10.1017/s0140525x10000725

1143 Henrich, J., Heine, S. J. \& Norenzayan, A. (2010b). Most people are not WEIRD. Nature, 466(7302), 29-29. https://doi.org/10.1038/466029a

1145 Henrich, J., Heine, S. J. \& Norenzayan, A. (2010c). The weirdest people in the world? 1146 Behavioral and Brain Sciences, 33(2), 61-83. https://doi.org/10.1017/S0140525X0999152X

1148 Johnson, C. P. \& Myers, S. M. (2007). Identification and evaluation of children with autism spectrum disorders. Pediatrics, 120(5), 1183-1215. https://doi.org/10.1542/peds.2007-2361

1151 Jones, R. \& Pitt, N. (1999). Health surveys in the workplace: Comparison of postal, email and world wide web methods. Occupational Medicine, 49(8), 556-558.

1154 Kaplowitz, M. D., Hadlock, T. D. \& Levine, R. (2004). A comparison of web and mail survey response rates. Public Opinion Quarterly, 68(1), 94-101. https://doi.org/10.1093/poq/nfh006

1157 Kinder und digitale medien. (2021). Retrieved August 23, 2021, from https://www.mmi.ch/de-ch/forschung/f\%C3\%BCr-studienteilnehmende/kidim

1159 Kirchhoff, C. \& Fuchs, C. (n.d.). Early childhood behavior questionnaire (ECBQ)

1160 fragebogen zum verhalten im kleinkindalter. Universitätsklinikum Ulm.

1161 https://research.bowdoin.edu/rothbart-temperament-questionnaires/instrument1162 descriptions/the-childrens-behavior-questionnaire/

1163 Koglin, U. \& Petermann, F. (2007). German version of the CBQ very short form. Universität Bremen. 
1165 Kretch, K. S., Franchak, J. M. \& Adolph, K. E. (2014). Crawling and walking infants see the world differently. Child Development, 85(4), 1503-1518. https://doi.org/10.1111/cdev.12206

1168 Kristen, S., Eisenbeis, H., Thoermer, C. \& Sodian, B. (2007). Temperamentsfragebogen 1169 für babys- revidierte form.

1170 Lafortune, F., Dery, M. \& Verlaan, P. (n.d.). Questionnaire sur les comportements des enfants - questionnaire court.

1172 Lange, B. P., Euler, H. A. \& Zaretsky, E. (2016). Sex differences in language competence of 3- to 6-year-old children. Applied Psycholinguistics, 37(6),

1175 LaRose, R. \& Tsai, H.-y. S. (2014). Completion rates and non-response error in online surveys: Comparing sweepstakes and pre-paid cash incentives in studies of online behavior. Computers in Human Behavior, 34, 110-119.

1179 Legare, C. H. \& Harris, P. L. (2016). The ontogeny of cultural learning. Child Development, 87(3), 633-642. https://doi.org/10.1111/cdev.12542

1181 Lisi, A. V. M.-D., Lisi, A. M.-D. \& Lisi, R. D. (2002). Biology, society, and behavior: The development of sex differences in cognition. Greenwood Publishing Group.

1183 LoBue, V. \& Adolph, K. E. (2019). Fear in infancy: Lessons from snakes, spiders,

1184 heights, and strangers. Developmental Psychology, 55(9), 1889-1907. https://doi.org/10.1037/dev0000675

1186 LoBue, V., Reider, L. B., Kim, E., Burris, J. L., Oleas, D. S., Buss, K. A.,

1187 Pérez-Edgar, K. \& Field, A. P. (2020). The importance of using multiple

1188 outcome measures in infant research. Infancy, 25 (4), 420-437.

1189 https://doi.org/10.1111/infa.12339

1190 Lüke, C., Grimminger, A., Rohlfing, K. J., Liszkowski, U. \& Ritterfeld, U. (2017). In 1191 infants' hands: Identification of preverbal infants at risk for primary language delay. Child Development, 88(2), 484-492. https://doi.org/10.1111/cdev.12610 
1193 Majnemer, A. \& Rosenblatt, B. (1994). Reliability of parental recall of developmental 1194 milestones. Pediatric Neurology, 10(4), 304-308. https://doi.org/10.1016/0887-8994(94)90126-0

1196 Manfreda, K. L., Bosnjak, M., Berzelak, J., Haas, I. \& Vehovar, V. (2008). Web surveys versus other survey modes: A meta-analysis comparing response rates. International Journal of Market Research, 50(1), 79-104. https://doi.org/10.1177/147078530805000107

1200 Mari, G. \& Keizer, R. (2020). Parental job loss and early child development in the great 1201 recession. https://doi.org/10.31235/osf.io/2596e

1202 Marian, V., Blumenfeld, H. K. \& Kaushanskaya, M. (2007). The language experience and proficiency questionnaire (LEAP-q): Assessing language profiles in bilinguals and multilinguals. Journal of Speech, Language, and Hearing Research, 50(4), 940-967. https://doi.org/10.1044/1092-4388(2007/067) https://research.bowdoin.edu/rothbart-temperament-questionnaires/instrumentdescriptions/the-childrens-behavior-questionnaire/

1209 Mayor, J. \& Mani, N. (2019). A short version of the MacArthur-bates communicative development inventories with high validity. Behavior Research Methods, 51(5),

1212 McGraw, M. B. (1943). The neuromuscular maturation of the human infant. Columbia University Press.

1214 Mclanahan, S. (2004). Diverging destinies: How children are faring under the second 1215 demographic transition. Demography, 41(4), 607-627.

1216 https://doi.org/10.1353/dem.2004.0033

1217 Miller, D. I. \& Halpern, D. F. (2014). The new science of cognitive sex differences.

1218 Trends in Cognitive Sciences, 18(1), 37-45. 
1220 Miller, L. E., Perkins, K. A., Dai, Y. G. \& Fein, D. A. (2017). Comparison of parent report and direct assessment of child skills in toddlers. Research in Autism Spectrum Disorders, 41-42, 57-65. https://doi.org/10.1016/j.rasd.2017.08.002

1223 Möhring, W. \& Frick, A. (2013). Touching up mental rotation: Effects of manual experience on 6-month-old infants' mental object rotation. Child Development,

1226 Montirosso, R., Cozzi, P., Putnam, S. P., Gartstein, M. A. \& Borgatti, R. (2011). Studying cross-cultural differences in temperament in the first year of life: United states and italy. International Journal of Behavioral Development, 35(1),

1230 Morales, M., Mundy, P., Crowson, M. M., Neal, A. R. \& Delgado, C. E. F. (2005). 27-37. https://doi.org/10.1177/0165025410368944

1234 Morris, A. S., Robinson, L. R. \& Eisenberg, N. (2006). Applying a multimethod perspective to the study of developmental psychology. In M. Eid \& E. Diener (Eds.), Handbook of multimethod measurment in psychology. American Psychological Association.

1238 Natalucci, G., Reinelt, T., Koller, B. M. \& Suppiger, D. (2021). Long-term effects of 1239 early nutrition on child development (LEARN). Retrieved August 23, 2021, from 1240 ㄴ https://www.usz.ch/fachbereich/neonatologie/forschung/ngnresearchcenter/current-research-projects/

1242 Nielsen, M., Haun, D. B. M., Kärtner, J. \& Legare, C. H. (2017). The persistent sampling bias in developmental psychology: A call to action. Journal of

1244 Experimental Child Psychology, 162, 31-38. https://doi.org/10.1016/j.jecp.2017.04.017

1246 Nielsen, M. \& Haun, D. (2016). Why developmental psychology is incomplete without comparative and cross-cultural perspectives. Philosophical Transactions of the 
1248

1249

1250 Nikolaizig, F. (2007). Temperamentsfragebogen für kinder [german translation of the

1251

1252

1253

1254 Nordahl-Hansen, A., Kaale, A. \& Ulvund, S. E. (2014). Language assessment in children 1255

1256

1257

1258

1259

1260

1261

1262

1263

1264

1265

1266 Powell, B., Steelman, L. C. \& Carini, R. M. (2006). Advancing age, advantaged youth:

1267

1268

1269

1270

1271

1272

1273 Putnam, S. P., Helbig, A. L., Gartstein, M. A., Rothbart, M. K. \& Leerkes, E. J.

1274

1275

1276 with autism spectrum disorder: Concurrent validity between report-based assessments and direct tests. Research in Autism Spectrum Disorders, 8(9), 1100-1106. https://doi.org/10.1016/j.rasd.2014.05.017

Pediatrics, A. A. o. (1992). American academy of pediatrics task force on infant positioning and SIDS. Pediatrics, 89, 1120-1126.

Pedlow, R., Sanson, A., Prior, M. \& Oberklaid, F. (1993). Stability of maternally reported temperament from infancy to 8 years. Developmental Psychology, 29(6), 998-1007. https://doi.org/10.1037/0012-1649.29.6.998

Peters-Martin, P. \& Wachs, T. D. (1984). A longitudinal study of temperament and its correlates in the first 12 months. Infant Behavior and Development, 7(3), 285-298. https://doi.org/10.1016/S0163-6383(84)80044-2 Parental age and the transmission of resources to children. Social Forces, 84(3), 1359-1390. https://doi.org/10.1353/sof.2006.0064

Putnam, S. P., Gartstein, M. A. \& Rothbart, M. K. (2006). Measurement of fine-grained aspects of toddler temperament: The early childhood behavior questionnaire. Infant Behavior and Development, 29(3), 386-401. https://doi.org/10.1016/j.infbeh.2006.01.004 (2014). Development and assessment of short and very short forms of the infant behavior questionnaire-revised. Journal of Persality Assessment, 96(4), 445-458. https://doi.org/10.1080/00223891.2013.841171 
1277 Putnam, S. P., Jacobs, J., Gartstein, M. A. \& Rothbart, M. K. (2010). Development 1278 and assessment of short and very short forms of the early childhood behavior

1280 Putnam, S. P. \& Rothbart, M. K. (2006). Development of short and very short forms of 1281 the children's behavior questionnaire. Journal of Personality Assessment, 87(1),

1283 Rege, M., Telle, K. \& Votruba, M. (2011). Parental job loss and children's school 1284 performance. The Review of Economic Studies, 78(4), 1462-1489.

1285 https://doi.org/10.1093/restud/rdr002

1286 Reilly, D., Neumann, D. L. \& Andrews, G. (2019). Gender differences in reading and 1287 writing achievement: Evidence from the national assessment of educational 1288 progress (NAEP). American Psychologist, 74(4), 445.

1289 Reips, U.-D. (2002). Standards for internet-based experimenting. Experimental Psychology, 49(4), 243-256. https://doi.org/10.1026//1618-3169.49.4.243

1291 Rhonda Folio, M. \& Fewell, R. R. (2000). Peabody developmental motor scales, 2nd 1292 edition. PRO-ED, Inc.

1293 Ross, C. E. \& Mirowsky, J. (1999). Parental divorce, life-course disruption, and adult 1294 depression. Journal of Marriage and Family, 61 (4), 1034-1045. https://doi.org/10.2307/354022

1296 Rothbart, M. K. (1981). Measurement of temperament in infancy. Child Development, 1297 52, 569-578. https://doi.org/10.2307/1129176

1298 Rothbart, M. K., Ahadi, S. A., Hershey, K. L. \& Fisher, P. (2001). Investigations of 1299 temperament at three to seven years: The children's behavior questionnaire. 1300 Child Development, 72(5), 1394-1408. https://doi.org/10.1111/1467-8624.00355

1301 Rothbart, M. K., Derryberry, D. \& Hershey, K. (2000). Stability of temperament in 1302 childhood: Laboratory infant assessment to parent report at seven years. In 1303 V. J. Molfese \& D. L. Molfese (Eds.), Temperament and personality development 1304 across the life span (pp. 85-119). Erlbaum. 
1305 Rubin, K. H., Burgess, K. B. \& Hastings, P. D. (2002). Stability and social-behavioral 1306 consequences of toddlers' inhibited temperament and parenting behaviors. Child Development, 73(2), 483-495. https://doi.org/10.1111/1467-8624.00419

1308 Sachse, S. \& Suchodoletz, W. v. (2008). Early identification of language delay by direct 1309 language assessment or parent report? Journal of Developmental $\&$ Behavioral Pediatrics, 29(1), 34-41. https://doi.org/10.1097/DBP.0b013e318146902a

1311 Sax, L. J., Gilmartin, S. K. \& Bryant, A. N. (2003). Assessing response rates and nonresponse bias in web and paper surveys. Research in Higher Education, 44(4), 409-432. https://doi.org/10.1023/A:1024232915870

1314 Schwarzer, G., Freitag, C., Buckel, R. \& Lofruthe, A. (2013). Crawling is associated with mental rotation ability by 9-month-old infants. Infancy, 18(3), 432-441. https://doi.org/10.1111/j.1532-7078.2012.00132.x

1317 Shih, T.-H. \& Fan, X. (2008). Comparing response rates from web and mail surveys: A meta-analysis. Field Methods, 20(3), 249-271. https://doi.org/10.1177/1525822X08317085

1320 Siegmüller, J., Kauschke, C., von Minnen, S. \& Bittner, D. (2011). Test zum satzverstehen von kindern. Elsevier.

1322 Smith, L. B. \& Thelen, E. (2003). Development as a dynamic system. Trends in Cognitive Sciences, 7(8), 343-348. https://doi.org/10.1016/S1364-6613(03)00156-6

1325 Soska, K. C., Robinson, S. R. \& Adolph, K. E. (2015). A new twist on old ideas: How sitting reorients crawlers. Developmental Science, 18(2), 206-218.

1328 Spencer, J. P., Vereijken, B., Diedrich, F. J. \& Thelen, E. (2000). Posture and the emergence of manual skills. Developmental Science, 3(2), 216-233. https://doi.org/10.1111/1467-7687.00115

1331 Statistik, B. f. (2020). Reproduktive Gesundheit. Retrieved January 22, 2020, from 1332 https://www.bfs.admin.ch/bfs/de/home/statistiken/gesundheit/ gesundheitszustand/reproduktive.html 
1334 Stevens, A. H. \& Schaller, J. (2011). Short-run effects of parental job loss on children's 1335 academic achievement. Economics of Education Review, 30(2), 289-299. https://doi.org/10.1016/j.econedurev.2010.10.002

1337 Stolt, S., Haataja, L., Lapinleimu, H. \& Lehtonen, L. (2008). Early lexical development 1338 of finnish children: A longitudinal study. First Language, 28(3), 259-279. https://doi.org/10.1177/0142723708091051

1340 Tahiroglu, D., Moses, L. J., Carlson, S. M., Mahy, C. E., Olofson, E. L. \& Sabbagh, M. A. (2014). The children's social understanding scale: Construction and validation of a parent-report measure for assessing individual differences in children's theories of mind. Developmental Psychology, 50(11), 2485-2497.

1345 Thomas, A. \& Chess, S. (1977). Temperament and development. Brunner/Mazel.

1346 van Schaik, C. P. \& Burkart, J. M. (2011). Social learning and evolution: The cultural intelligence hypothesis. Philosophical Transactions of the Royal Society B: Biological Sciences, 366(1567), 1008-1016. https://doi.org/10.1098/rstb.2010.0304

1350 Vonderlin, E., Ropeter, A. \& Pauen, S. (2012). Erfassung des frühkindlichen temperaments mit dem infant behavior questionnaire revised. Zeitschrift für Kinder- und Jugendpsychiatrie und Psychotherapie, 40(5), 307-314. https://doi.org/10.1024/1422-4917/a000187

1354 Vygotsky, L. S. (1978). Mind and society: The development of higher mental processes. $1355 \quad$ Harvard University Press.

1356 Wellman, H. M. (2007). Understanding the psychological world: Developing a theory of mind. Blackwell handbook of childhood cognitive development (pp. 167-187).

1358 John Wiley \& Sons, Ltd. Retrieved May 19, 2020, from https://onlinelibrary.wiley.com/doi/abs/10.1002/9780470996652.ch8

1360 Wohlwill, J. F. (1973). The study of behavioral development. Academic Press. 
1361 Yeung, W. J., Linver, M. R. \& Brooks-Gunn, J. (2002). How money matters for young $1362 \quad$ children's development: Parental investment and family processes. Child 1363 Development, 73(6), 1861-1879. https://doi.org/10.1111/1467-8624.t01-1-00511 1364 Zelazo, P. R. (1998). McGraw and the development of unaided walking. Developmental 1365 Review, 18(4), 449-471. https://doi.org/10.1006/drev.1997.0460

1366 Zwickel, J. (2009). Agency attribution and visuospatial perspective taking. Psychonomic 1367 Bulletin $\&$ Review, 16(6), 1089-1093. https://doi.org/10.3758/PBR.16.6.1089 


\section{Appendix}

\section{Details on Scales}

In the following sections, we describe the answer options of the cognitive,

1371 language, motor, and social-emotional scale in more detail and give examples of answer 1372 options and items of each scale. All the items of all scales are available on

1373 https://osf.io/ar7xp/.

\section{Cognitive Scale}

Answer options. Caregivers are asked to indicate whether or not they

1376 observe the behaviour in question. We display answer options in a dichotomous manner.

1377 The positive option is "Yes" and, to be unambiguous, relevant factors of the respective

1378 behaviour are additionally outlined concisely for some items. Hence, the answer options

1379 include exact success criteria which have to be met (e.g., counting 3 or more objects).

1380 The negative option is "No" and, where necessary, alternative behaviour which would

1381 not justify a positive choice is sketched (e.g., counting less than 3 objects). As in the

1382 questions for the motor scale, if caregivers indicate "Yes", they are asked to additionally

1383 indicate since when the child mastered the skill, see Figure 1 .

1384 Exemplary items. To illustrate, we exemplarily describe one item for each 1385 construct. An early sensori-motor item on exploration behaviour asks: "Does your 1386 child play with or examine his/her fingers?". Caregivers can answer with either "Yes,

1387 when my child is calmly lying on his/her back, my child plays with his/her hands or 1388 examines them (turning them around, opening and closing them)" or "No". One of the 1389 first Problem-solving items assesses means-end behaviour: "Does your child pull at flat 1390 objects (e.g., a cloth or blanket) to grasp a toy that is lying on it?". This item has the 1391 answer alternatives: "Yes" or "No, my child moves towards the toy". A Numerical and 1392 categorical knowledge item investigates children's counting abilities: "Can your child 1393 count out 10 objects (e.g., building blocks)?". Caregivers can answer with "Yes, my 1394 child says "one" to the first object, "two" to the second and "three" to the third, etc." 1395 or "No". 


\section{Language Scale}

Answer options. For each sentence of the morphological and syntactical

1398 items, caregivers are instructed to indicate how often their child produces such or a

1399 similar sentence. The questions can be answered via a 5-point Likert scale (never; less

1400 than once a week; at least once a week, but not every day; once or twice a day; more

1401 than twice a day) similar to the Children's Communication Checklist - 2 (CCC2,

1402 Bishop, 2003).

1403 Exemplary items. To illustrate, we provide some examples for items and

1404 prototypical sentences. An item for Gestures asks for: "Does [Child's Name] point at

1405 objects that [Child's Name] wants or would like to show you?". An item for Morphology

1406 assesses one of the plural forms in Swiss German like this: "Holsch üsi Jackene?"

1407 (Engl. "Can you bring our jackets?"). An item for Syntax conjunctions includes the

1408 following sentence: "Wämmer wiiterspile oder wetsch es Buech aluege?" (Engl. "Do we

1409 want to keep playing or should we look at a book?").

\section{Motor Scale}

1411 Answer options. For each item a dichotomous scale is used with which the

1412 caregivers are asked to indicate whether their child has attained the skill (Yes) or not

1413 (No). If caregivers indicate "Yes", they are further asked to indicate since when the

1414 child mastered the skill, see Figure 1.

1415 Exemplary items. To illustrate, we provide some examples for typical items.

1416 Visual-motor integration items assess eye-hand coordination with objects: "My child

1417 can stack 5 building blocks" and without objects: "My child can clap his/her hands".

1418 Specific Grasping items assess the way objects are grasped and held how a child uses

1419 his/her hands for grasping: "My child can grasp a rattle, lying on his/her back".

1420 Graphomotorics items assess how the child uses pencils to draw and write: "My child

1421 imitates me drawing a line". Stationary motor skills items assess body posture: "When

1422 I place my child on his/her tummy and shake a rattle, he/she rotates his head to locate

1423 the rattle". Locomotion items assess movement in space: "My child can roll from back 
1424 to tummy". Object manipulation items assess the manipulation of a ball: "When I sit on

1425 the floor with my child and roll a ball towards him/her, he/she will roll the ball back to 1426 me".

\section{Social-Emotional Scale}

1428 Details on questionnaires. In the following sections, we provide additional 1429 information of the established questionnaires, which are used to assess children's 1430 social-emotional development in the APP.

1431 Infant Behavior Questionnaire - Revised (IBQ-R). The original IBQ 1432 was developed by Rothbart (1981) and revised as IBQ-R by Gartstein and Rothbart 1433 (2003). The full version of the IBQ-R includes 14 scales and 191 items. For feasibility 1434 and efficiency reasons, we implemented the Very Short Form version (Putnam et al., 1435 2014, IBQ-R-VSF, ) that includes 37 items in 3 broad scales (Positive

1436 Affectivity/Surgency, Negative Emotionality, Orienting/Regulatory Capacity). For the 1437 non-English versions, we used the available translations (German: Kristen et al., 2007 1438 and slightly revised by Vonderlin et al., 2012; Italian: Montirosso et al., 2011; French: 1439 Cascales, 2011).

1440 Early Childhood Behavior Questionnaire (ECBQ). The ECBQ was 1441 developed by Putnam et al. (2006). The original questionnaire includes 18 scales and 1442201 items. We used the very short form version (Putnam et al., 2010) that includes 36 1443 items in 3 scales (similar to the IBQ-R-VSF: Positive Affectivity/Surgency, Negative 1444 Emotionality, Orienting/Regulatory Capacity). For the non-English versions, we used 1445 the available translations (German: fuchs_early_nodate; Kirchhoff and Fuchs, n.d. 1446 French: Goupil, n.d.; Italian: Cozzi et al., 2013 ).

1447 Children's Behavior Questionnaire (CBQ). The CBQ was developed by 1448 Rothbart et al. (2001). The original questionnaire includes 15 scales and 195 items. We 1449 used the very short form version (Putnam \& Rothbart, 2006) that includes 36 items and 14503 scales (similar as in the IBQ-R-VSF: Surgency, Negative Affect, and Effortful 1451 Control). For the non-English versions, we used the available translations (German: 
1452 Koglin and Petermann, 2007; Nikolaizig, 2007; French: Lafortune et al., n.d., Italian:

1453 Matricardi, n.d.).

1454 Children's Social Understanding Scale (CSUS). Children's ToM is

1455 usually measured via a range of laboratory paradigms that assess the understanding of

1456 mental states such as beliefs, desires, emotions, and intentions (e.g., Wellman, 2007).

1457 We implemented the CSUS (Tahiroglu et al., 2014), a caregiver-report ToM measure. It

1458 includes 6 scales (belief, knowledge, perception, desire, intention, emotion) with a total

1459 of 42 items which have been shown to be a measure of individual differences in children's

1460 ToM with very high internal consistency, test-retest reliability, and predictive validity.

1461 Answer options. The items used in the different scales describe behaviour

1462 that frequently occur in everyday contexts (e.g., "When being dressed or undressed,",

1463 "When playing outdoors", "When told no"). Accordingly, caregivers are asked to

1464 indicate how often their child showed a particular behaviour during the last week (i.e.,

1465 the past seven days) on a 7-point Likert-style format ranging from "never" to "always".

1466 We applied the same scales as used in the original questionnaires. 


\section{Equation used for analyses of psychometric properties}

1468 To analyse the objectivity and criterion validity of the APP, we used different 1469 multi-level logistic regressions predicting either the AoA for the motor and cognitive 1470 items or the language scale index for language skills by domain (motor or cognition), 1471 caregiver education (mother and father), caregiver age (mother and father), app user

1472 (mother or father), sex of the child, and pregnancy week in which the child was born 1.

$$
\begin{array}{r}
\text { AoA/Language Index } \sim \\
\text { Domain }+ \text { Education }_{\text {Father }}+\text { Education }_{\text {Mother }}+ \\
\text { Age }_{\text {Father }}+\text { Age }_{\text {Mother }}+ \\
\mathrm{APP}_{\text {User }}+\text { Sex }_{\text {Child }}+\text { PregnancyWeek }_{\text {Child }}+(1 \mid \text { Item })
\end{array}
$$




\section{Table A1}

Internal Consistency (Cronbach's $\alpha$ ) for Cognition, Language, Fine Motor, and Gross Motor Items for Different Age Ranges.

\begin{tabular}{|c|c|c|}
\hline Scale & Age Range (Months) & $\alpha$ \\
\hline Cognition & $3-6$ & 0.81 \\
\hline Cognition & $6-12$ & 0.449 \\
\hline Cognition & $12-18$ & 0.424 \\
\hline Cognition & $18-24$ & 0.421 \\
\hline Cognition & $24-36$ & 0.575 \\
\hline Cognition & $36-48$ & 0.334 \\
\hline Language & $24-36$ & 0.985 \\
\hline Language & $36-48$ & 0.982 \\
\hline Language & $48-72$ & 0.982 \\
\hline Fine Motor & $3-6$ & 0.918 \\
\hline Fine Motor & $6-12$ & 0.831 \\
\hline Fine Motor & $12-18$ & 0.653 \\
\hline Fine Motor & $18-30$ & 0.742 \\
\hline Fine Motor & $30-44$ & 0.817 \\
\hline Fine Motor & $44-72$ & 0.835 \\
\hline Gross Motor & $3-6$ & 0.9 \\
\hline Gross Motor & $6-12$ & 0.889 \\
\hline Gross Motor & $12-18$ & 0.738 \\
\hline Gross Motor & $18-30$ & 0.748 \\
\hline Gross Motor & $30-44$ & 0.755 \\
\hline Gross Motor & $44-72$ & 0.812 \\
\hline
\end{tabular}


Table A2

Number of Users (Total, Mothers, and Fathers) of the 15 Countries with the Largest Numbers of Users.

\begin{tabular}{lrrr}
\hline Country & Total & $n$ (Mothers) & $n$ (Fathers) \\
\hline Switzerland & 2472 & 1227 & 1245 \\
Germany & 1827 & 918 & 909 \\
Austria & 68 & 35 & 33 \\
Italy & 52 & 31 & 21 \\
Poland & 35 & 12 & 23 \\
United States & 23 & 12 & 11 \\
Turkey & 22 & 14 & 8 \\
Russia & 21 & 9 & 12 \\
United Kingdom & 18 & 15 & 3 \\
Romania & 18 & 8 & 12 \\
Spain & 18 & 12 & 6 \\
Bosnia & 17 & 6 & 11 \\
France & 15 & 9 & 6 \\
Kazakhstan & 15 & 2 & 13 \\
Kosovo & 13 & & 6 \\
\hline & & 9 & \\
\hline
\end{tabular}

